\title{
Cross-cultural data shows musical scales evolved to maximise imperfect fifths
}

\author{
John M. McBride ${ }^{1, *}$ and Tsvi Tlusty ${ }^{1,2, *}$ \\ ${ }^{1}$ Center for Soft and Living Matter, Institute for Basic Science, Ulsan 44919, South Korea \\ ${ }^{2}$ Department of Physics, Ulsan National Institute of Science and Technology, Ulsan 44919, South Korea \\ *jmmcbride@protonmail.com, tsvitlusty@gmail.com
}

June 1, 2020

Musical scales are used throughout the world, but the question of how they evolved remains open. Some suggest that scales based on the harmonic series are inherently pleasant, while others propose that scales are chosen that are easy to communicate. However, testing these theories has been hindered by the sparseness of empirical evidence. Here, we assimilate data from diverse ethnomusicological sources into a cross-cultural database of scales. We generate populations of scales based on multiple theories and assess their similarity to empirical distributions from the database. Most scales tend to include intervals which are close in size to perfect fifths ("imperfect fifths"), and packing arguments explain the salient features of the distributions. Scales are also preferred if their intervals are compressible, which may facilitate efficient communication and memory of melodies. While scales appear to evolve according to various selection pressures, the simplest, imperfect-fifths packing model best fits the empirical data.

How and why did humans evolve to create and appreciate music? The question arose at the dawn of evolutionary theory and been asked ever since 1 14. Studying musical features that are conserved across cultures may lead to possible answers. 5, 6]. Two such universal features are the use of discrete pitches and the octave, defined as an interval between two notes where one note is double the frequency of the other 7 . Taken together, these form the musical scale, defined as a set of intervals spanning an octave (Fig. 11A). Musical scales can therefore be considered solutions to the problem of partitioning an octave into intervals, and thus can be treated mathematically. Examination of scales from different cultures can help elucidate the basic perception and production mechanisms that humans share and shed light on this evolutionary puzzle.

One theory on the origin of scales suggests that the frequency ratios of intervals in a scale ought to consist of simple integers [8]. After the octave (2:1), the simplest ratio is $3: 2$, referred to in Western musical theory as a perfect fifth. Frequencies related by simple integer ratios naturally occur in the harmonic series - a plucked string will produce a complex sound with a fundamental frequency accompanied by integer multiples of the fundamental. The theory that scales are related to harmonicity follows from the idea that exposure to harmonic sounds in animal vocalisations may have conditioned humans to respond positively to them 9] 10. Musical features related to harmonicity - harmonic intervals [11-13, octave equivalence [14, 15, a link between consonance and harmonicity 16 18 are indeed widespread, although their universality is disputed [19, 20. One study attempted to explain the origin of scales as maximization of harmonicity 8, however the universality of their findings is limited by the scope of cultures considered 21].

The vocal mistuning theory states that scales, and intervals, were chosen not due to harmonicity, but because they were easy to communicate 22,23 . We perceive intervals as categories [24 27, and due to errors in producing [22, 28, 29], and perceiving notes $30-34$, musical intervals are not exact frequency ratios, but rather they span a range of acceptable interval sizes. Any overlap between interval categories will then result in errors in transmission. This theory appears promising, but it has not yet been rigorously investigated.

These two theories were proposed separately, yet they are not mutually exclusive. In this paper, we modify, integrate and expand upon these ideas to construct a general, stochastic model which generates populations of scales. Our aim is to test which model best mimics scales created by humans. To this end, we assembled the most diverse and extensive database of scales from ethnomusicological records. By comparing modelgenerated theoretical distributions with the empirical distributions, we find that the theory that best fits the data is the simplest. Most scales are arranged to maximise inclusion of imperfect fifths - perfect fifths with a tolerance for error. Scales are often found to be compressible, which may make them easier to transmit. Adding more detail, beyond fifths, to harmonicity-based theories decreased their performance, which suggests that only the first few harmonics are significant in this context.

\section{Results}

\section{Harmonicity Models}

The main assumption underpinning the harmonicity theory is that human pitch processing evolved to take advantage of nat- 
ural harmonic sounds 37. For example, harmonic amplitudes typically decay with harmonic number 9 15, and correspondingly, lower harmonics tend to be more dominant in pitch perception 38. Thus many proxy measures of harmonicity contain parameters to account for harmonic decay [39]. However, to minimize a priori assumptions and model parameters, we avoid explicitly modelling harmonic decay. Instead, we test two simple harmonicity theories which differ in how they treat higher order harmonics.

The first harmonicity model (HAR), assumes that there is no harmonic decay, for which the model of reference 8 is appropriate. This model scores an interval $f_{2} / f_{1}$ defined by two frequencies, $f_{1}$ and $f_{2}$, based on the fraction of harmonics of $f_{2}$ that are matched with the harmonics of $f_{1}$ in an infinite series. Humans do not notice small deviations from simple ratios 40 42], and the model accounts for this by considering intervals as categories of width $w$ cents; intervals are measured in cents such that an octave is $1200 \log _{2} \frac{f_{2}}{f_{1}}=1200$ cents. Intervals are assigned to a category according to the highest scoring interval within $w / 2$ cents. The resulting template (Fig. 1B) is used to calculate the average harmonicity score for each scale across all $N \times(N-1)$ possible intervals, apart from the octave; $N$ is the number of notes in a scale. We make no assumptions about tonality, and thus all intervals are weighted equally. The HAR model assumes that scales evolved to maximise this harmonicity score.

The second harmonicity model (FIF) considers the limiting case of high harmonic decay. As harmonic decay increases, eventually a few intervals in a harmonic series become dominant (SI Table 1), in the order of unison, octave, fifth, etc. Thus, this model assumes that due to harmonic decay, only the octave and the fifth significantly affected the evolution of scales. Given this, we simply count the fraction of intervals that are fifths, out of all $N \times(N-1)$ possible intervals - we do not count the octave, and we make no assumptions about tonality. We allow a tolerance for errors, $w$, and thus define "imperfect fifths" as intervals of size $702 \pm w / 2$ cents. The FIF model assumes that scales evolved to maximise the number of imperfect fifths that can be formed in a scale.

\section{Transmittability Model}

The transmittability theory assumes that intervals are perceived as broad categories, and the humans make errors in both production and perception of intervals. Intervals must thus be large enough to avoid errors in transmission due overlapping interval categories (SI Fig. 1). This is not sufficient, however, to explain the considerable convergence in scales across cultures. We can further consider that scales are optimized for minimizing errors in transmission by favouring the use of large intervals, however this bias exclusively favours equidistant scales (SI pg. 4). While equidistant scales do exist 43 46, they are a minority [47, so additional mechanisms are needed to explain the origin of scales.

When humans encode continuous audiovisual information such as speech, musical rhythm, brightness, or color, there is evidence that it is done efficiently [48 54]. If the same is true for pitch, then compressible scales would facilitate com-

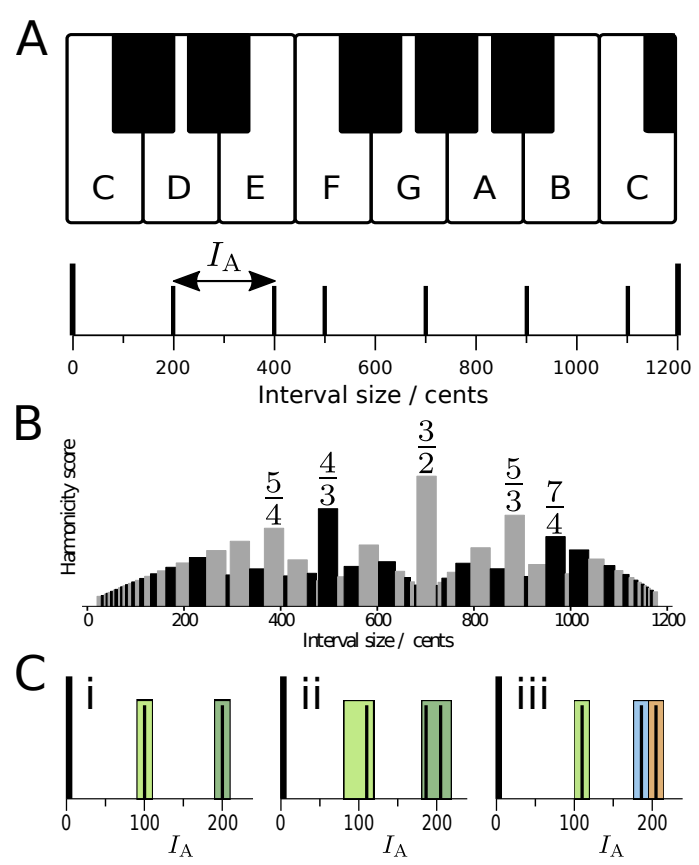

Figure 1: A: The major scale starting on a $\mathrm{C}$ shown on a piano (equal temperament), and as interval sizes (in cents) of each note measured from the first note. Intervals between adjacent pairs of notes are denoted by $I_{\mathrm{A}}$. B: We use a harmonicity template to assign harmonicity scores to intervals [8]. The highest scoring intervals (frequency ratios are shown for the top five) act as windows of attraction, whereby any interval in this window is assigned its score. In this case the maximum window size is $w=40$ cents. C: Adjacent interval, $I_{\mathrm{A}}$, sets for the major scale in equal temperament (i) and just intonation (ii \& iii). Boxes indicate interval categories, where the width represents the error, and boxes with similar colours are related by a common denominator: (i) is losslessly compressed; a similar compression of (ii) is lossy; (iii) is uncompressed and therefore costs more to transmit.

munication of melodies. An example of a compressible scale is the equal temperament major scale (Fig. 14). We can represent this scale using its notes $(\mathrm{C}, \mathrm{D}$, etc.) or as a sequence of adjacent intervals, $I_{\mathrm{A}}: 200,200,100,200,200,200,100$. The most compressed representation uses an alphabet size of one by encoding the large interval (200) in terms of the small one (100) (Fig. 1 Ci). However for the just intonation tuning $I_{\mathrm{A}}: 204,182,112,204,182,204,112$ - this code is lossy (Fig. 1 Cii). Lossless compression would require a three letter alphabet (Fig. 11Ciii).

With this in mind, we create a third model (TRANS) to test whether scales evolved to be compressible. We define scale compressibility as how accurately an $I_{\mathrm{A}}$ set can be represented by a simple interval category template. We consider templates 
with categories centred about integer multiples of a common denominator (e.g., 100, 200, 300). Accuracy in this case corresponds to the distance between $I_{\mathrm{A}}$ and the centre of the closest category. Note that this is merely an approximation of information-theoretic compressibility as we do not explicitly calculate the information content. The TRANS model assumes that scales are selected for their ease of communication, and that this is captured by our measure of compressibility.

\section{Generative Monte-Carlo simulations}

To test the theories, we use Monte Carlo simulations to generate populations of scales, based on the above discussion on harmonicity and transmittability. We impose a minimum interval size constraint, $I_{\min }$, such that scales with intervals $<I_{\min }$ are rejected. Depending on the theory, we accept or reject scales according to a cost function and a corresponding Boltzmann probability, which allows us to control the strength of the bias via a parameter $\beta$. As $\beta$ increases, the generated populations become increasingly selective and eventually too selective, resulting in an optimal $\beta$ at which the generated scales best matches the empirical scales.

We examine and compare results for five models:

- RAN: random scales subject to no constraints or biases.

- MIN: random scales with a minimal interval constraint.

- FIF: scales that maximize the number of imperfect fifths.

- HAR: scales biased to have high harmonic similarity score.

- TRANS: transmittable scales biased to be compressible.

To simplify our simulations, we assume that adjacent intervals, $I_{\mathrm{A}}$, in a scale add up to an octave of fixed size, 1200 cents. We fix the number of notes, $N$, as a model parameter. In addition, (i) The FIF, HAR, and TRANS models are also subject to the $I_{\min }$ constraint of the MIN model; (ii) The FIF and HAR models include the maximum window size $w$ as a variable parameter; (iii) The TRANS model has a single parameter, $n$, that affects the way inaccuracies due to compression are penalized.

For each model we generate a sample size of $S=10^{4}$ scales. Unless stated otherwise, we show results for $I_{\text {min }}=80$ cents, $w=20$ and $n=2$. We varied the parameters $I_{\min }, w$ and $n$, and found that differences are not negligible but do not alter the main results of this work (SI Fig. 2). We optimized the strength of the bias for each model and each $N$ by tuning $\beta$ (SI Table 4).

\section{Scale database}

To evaluate the models, we created a database with the aim of recording the diversity of scales used by different cultures. To this end, we compiled scales that use exact mathematical ratios for interval sizes (e.g., Western, Arabic, Carnatic), taking into account usage of different tuning systems (e.g., equal temperament, just intonation) [12. Despite the wealth of scales of this type, these cultures are but a fraction of the those that produce music. In our aim to obtain a comprehensive, diverse database we amalgamated work from ethnomusicologists who

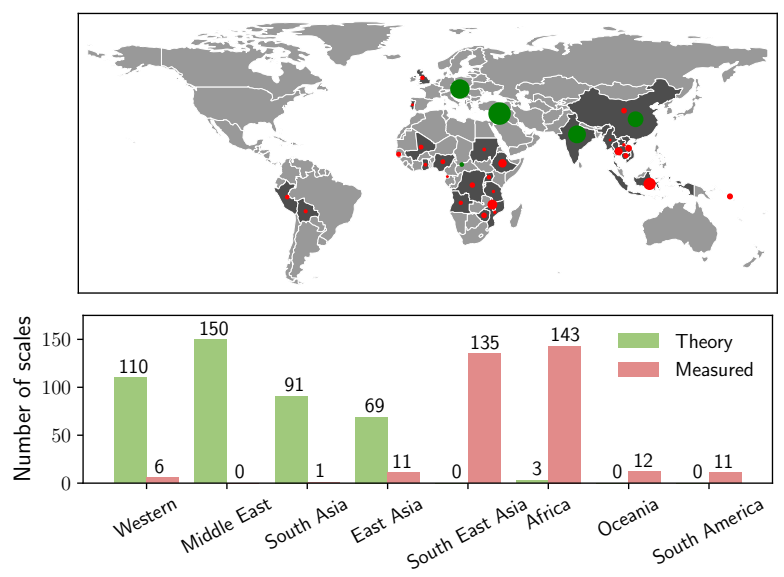

Figure 2: Scales in the database either come from a mathematical theory (theory) or from measurements from instruments or recordings (measured). The map shows the origin of the scales (theory: continents; measured: dark shaded countries), with sample size, $S$, indicated by the marker size.

measured tuning systems used across the world. The database is split into 'theory' scales (sample size, $S=423$ ) which have exact theoretical values for frequency ratios, and 'measured' scales $(S=319)$ which were inferred from measurements of instrument tunings and recorded performances (Fig. 2). 'Measured' scales within a culture can vary significantly (e.g., the Gamelan slendro scale), and given our goal of capturing this diversity, we recorded multiple 'measured' scales even if they have the same name. A full list of references, inclusion criteria, and the numbers/types of scales taken from each reference, are presented in the SI, Table 2 and 3.

A caveat with this approach is that the database might reflect hidden biases. For example, imperial dominance and globalization can result in homogenization of cultures [35, and many ethnomusicologists were biased towards reporting on cultures that were more distinct than similar [6]. It is therefore inherently difficult to rigorously define a 'correct' empirical distribution of scales, but we believe that this is a suitable approach to start with. Another issue is that despite the wide coverage of the database, it includes only a fraction of cultures, both geographically and historically, so it can be considered a lower bound on the diversity of scales. These limitations may be overcome with the aid of tools that can reliably estimate scales from ethnographic recordings, which will enable studies on a larger scale [28, 36. By making our database open we hope to inspire others to plug these gaps and undertake further quantitative analysis of musical scales. 


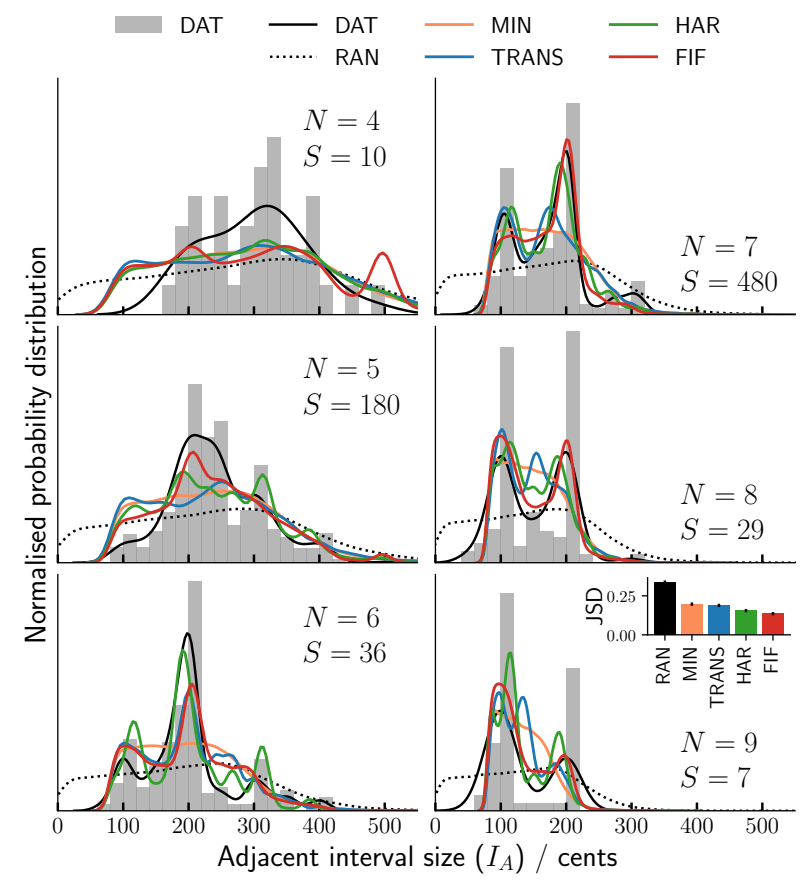

Figure 3: Adjacent interval $\left(I_{\mathrm{A}}\right)$ probability distributions for empirical DAT scales (black solid line) and model scales. The sum of the probabilities is one. Lines are fitted to histograms using kernel density estimation. Histograms (bin size is 20 cents) and sample sizes $(S)$ are shown only for DAT scales. Each panel shows the distributions for a given note number $N=4-9$. Inset: the average Jensen-Shannon divergence, JSD, between model and DAT distributions for each model; whiskers indicate $95 \%$ confidence intervals. eft (smaller interval sizes) and the range shrinks. This trend holds also for RAN scales, and by including the minimum interval constraint (MIN model), the ranges of the theoretically generated distributions approximate the empirical ones. This indicates that at the most basic level, the choice of intervals may be considered a packing problem. Given $N$ intervals of size $I_{\mathrm{A}}$, their distribution depends on the possible ways that these $N$ intervals can be combined into an octave. Still, this description fails to explain the significant peaks and troughs in the distributions.

The FIF model best replicates empirical adjacent interval distributions

The models which use biases result in distributions with clear peaks and troughs (Fig. 3). In the TRANS distributions, some of the peaks are aligned with the DAT distributions $(N=6,7)$, while others are clearly not $(N=8,9)$. The existence of prominent peaks at $1200 / N$, in addition to other peaks, indicates that the bias favours equidistant scales, but not exclusively. The HAR and FIF distributions match many of the features of the DAT distributions. The sample-size averaged JensenShannon divergence between the DAT and the model distributions (Fig. 3 inset, SI Table 5) show that the FIF model best fits the DAT distributions. This is somewhat unexpected, as the FIF model only specifies the inclusion of fifths, a large interval, while it predicts the distribution of smaller adjacent intervals. To explain the performance of the FIF model, we can consider how these building blocks are arranged into scales.

The FIF model best replicates the empirical note distributions

The MIN, and TRANS models accept or reject scales based only on their adjacent intervals, disregarding their order. Since the HAR and FIF models use all intervals (by comparing all pairs of notes) in their cost functions, they take the interval order into account. Hence, to see if interval order is indeed an important determinant, we examine how the notes are distributed in the scales (Fig. 4). Only $N=5$ and $N=7$ are shown due to the stricter need for sufficient statistics when considering the full scale distributions. The distributions of DAT scales show that certain notes are favoured, with notable peaks at 200, 500 and 700 cents for both $N$. Apart from exclusion zones at the boundaries, every note (when discretized in bins of width 30 cents) is used in at least one scale.

Among the models, the MIN distributions lack much detail beyond noise, apart from slight undulations with a periodicity of $\frac{1200}{N}$ cents. The TRANS distributions contain some notable peaks. Peaks at 500 and 700 cents fit the DAT distributions, while the 600 cents peak for $N=5$ is indubitably incorrect. The HAR and FIF distributions replicate the main features of the DAT distributions, including peaks, contour and troughs. The extra detail in the HAR model appears as additional peaks at harmonic intervals in the distributions.

Lacking rules for ordering intervals into scales, the 297 TRANS model still performs well

We can compactly represent the performance of models by two scalar measurables (Fig. 5): 

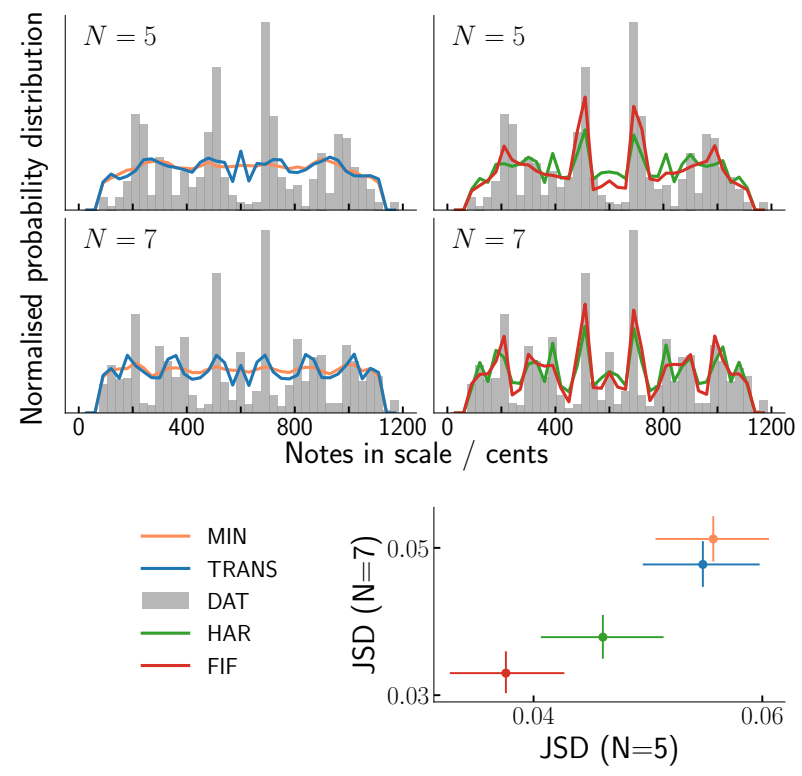

Figure 4: Top: Probability distribution of scale notes for DAT and model scales. Histograms are shown as lines for model results; sample sizes for DAT scales are the same as in Fig 3 Histograms (bin size is 30 cents) are truncated at 15 and 1185 cents for clarity. Bottom: JSD between model and DAT distributions for $N=5$ and $N=7$; whiskers indicate $95 \%$ confidence intervals.

(i) The sample-size-weighted Jensen-Shannon divergence between DAT and theoretical $I_{\mathrm{A}}$ distributions for each model.

(ii) $f_{\mathrm{D}}-$ The fraction of DAT scales that are found in the model-generated populations.

Due to our assumption that intervals in a scale add up to 1200 cents, many 'measured' scales cannot be found by the model due to inexact octave tunings, resulting in an upper bound of $f_{\mathrm{D}} \approx 0.8$. Additionally, the models generate samples of $S=10^{4}$ scales, and while JSD converges by this point, $f_{\mathrm{D}}$ does not - i.e., as $S$ increases, so will $f_{\mathrm{D}}$.

The TRANS model is better suited to predicting DAT scales than predicting DAT note distributions. This is surprising, given that the TRANS model offers no guidance on how to arrange intervals into a scale. Correlations between intervals in our database show that two intervals are usually ordered such that large goes with small and vice versa (we call this 'well-mixed'). Two small (large) intervals are half as likely to be placed together in DAT scales than random chance would predict (SI Fig. 3). By shuffling the model scales with a bias towards mixing of sizes, we observed an average increase in $f_{\mathrm{D}}$ of $12 \%$ for the TRANS model while seeing an average decrease of $22 \%$ for the FIF model (SI Fig. 4). So while scales tend to be arranged so that interval sizes are well-mixed, it is more important that they are arranged to maximise the number of fifths. Given that the FIF (HAR) and TRANS models are independent of each other, the performance of the TRANS model is quite notable.

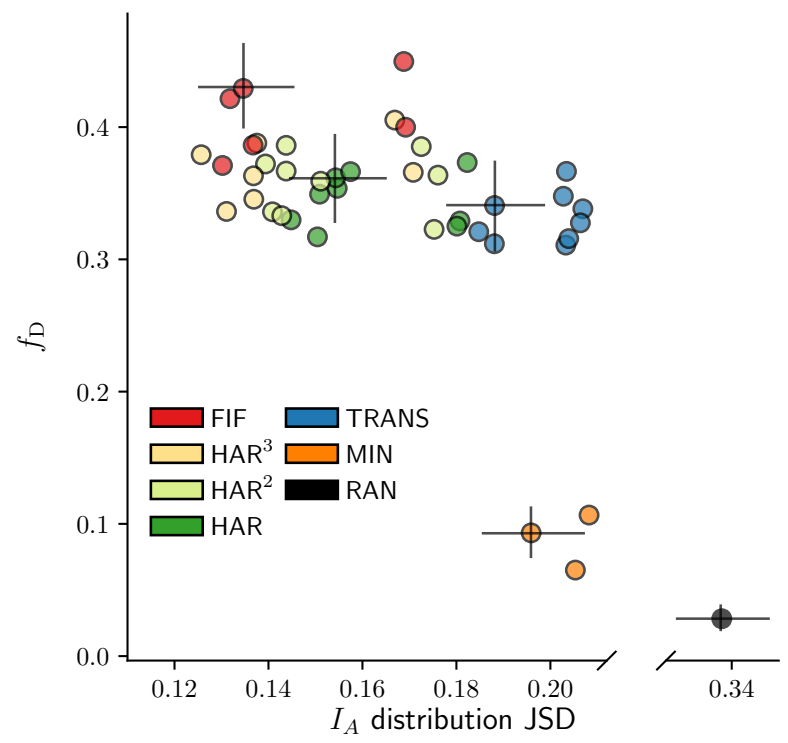

Figure 5: Model performance is plotted as the JensenShannon divergence (JSD) between the DAT and model $I_{\mathrm{A}}$ distributions against $f_{\mathrm{D}}$, the fraction of DAT scales predicted. Superior models exhibit low JSD values and high values of $f_{\mathrm{D}}$. Results for altered versions of the HAR model are also shown as $\mathrm{HAR}^{2}$ and $\mathrm{HAR}^{3}$. For each model, results are shown for combinations of parameters: $I_{\min }=[70,80,90]$, $w=[5,10,15,20], n=[1,2,3]$, and in each case the bias strength $\beta$ is optimized. Whiskers indicating $95 \%$ confidence intervals are only displayed for the models shown in Fig. 3 Fig. 4 and Fig. 6

\section{HAR model performance relies heavily on imperfect} fifths

Despite the FIF model being a much simpler version of the HAR model, the FIF model achieves superior results according to our metrics (Fig. 5]. To identify possible reasons, we interpolate between the HAR and FIF model templates by taking as a harmonicity score the $m$-th power of the harmonicity template in Fig. 1B, thereby approaching the FIF template as $m \rightarrow \infty$. Fig. 5 shows the results for models where we use $m=2\left(\mathrm{HAR}^{2}\right)$ and $m=3\left(\mathrm{HAR}^{3}\right)$, together with the original HAR model $(m=1)$. The results indicate that as one interpolates from the HAR model to the FIF model the performance also interpolates continuously. Additionally, we note that the FIF and HAR models are strongly correlated (e.g., 

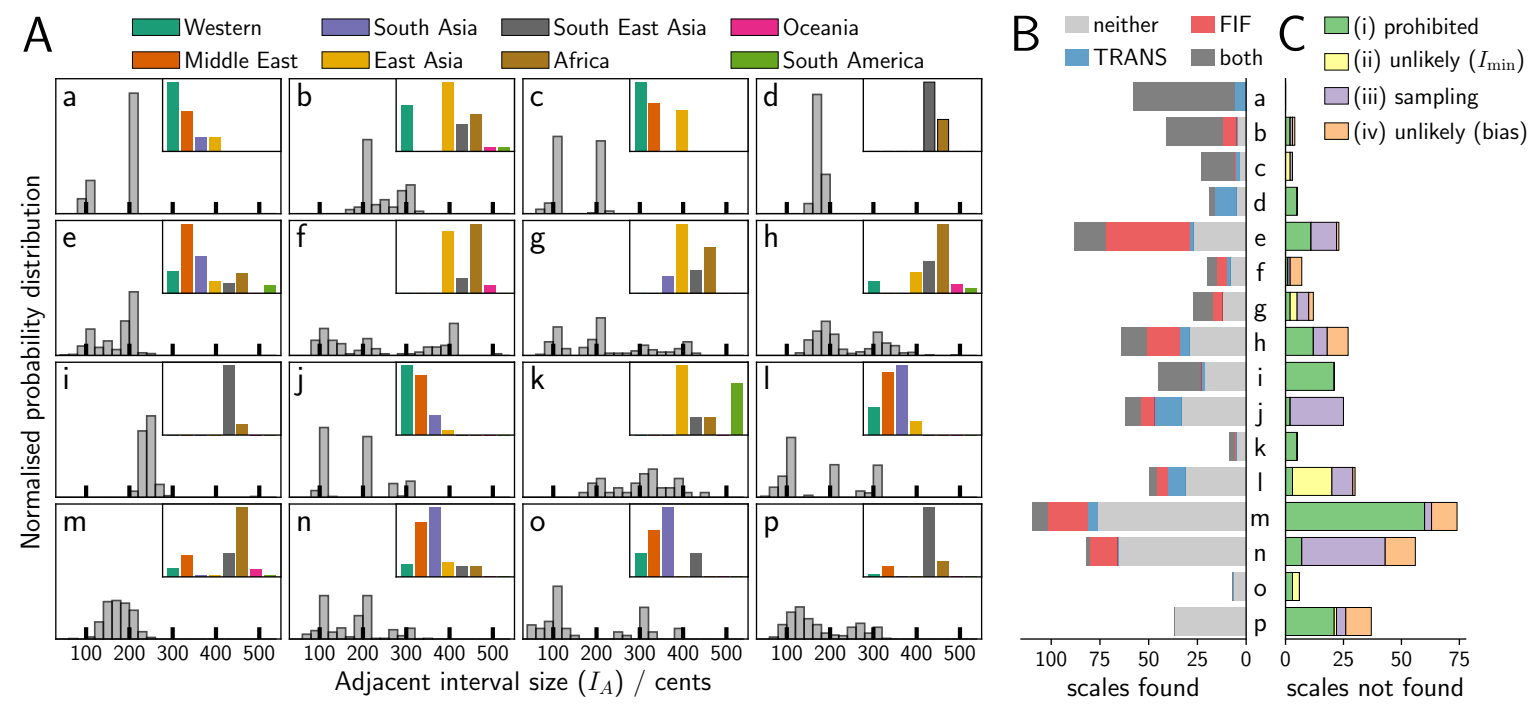

Figure 6: A: $I_{\mathrm{A}}$ interval distributions of empirical DAT scales for clusters a-p and their geographical composition (inset). B: Number of DAT scales found by neither model, only the TRANS model, only the FIF model, or both models, for cluster a-p. C: For the scales that are not found by any of the TRANS, FIF, or HAR models, we show the breakdown of the reasons why: (i) prohibited due to model constraints, (ii) unlikely due to model constraints, (iii) insufficient sampling, (iv) unlikely due to model biases.

Pearson's $r=0.76$ at $w=20$, SI Fig. 5B). This implies that fifths are a dominant part of the HAR model, while the extra detail merely hinders performance.

\section{Clustering of scales reveals differences between mode predictions}

To further understand how the models work, and in particular why two disparate theories (harmonicity and transmittability) perform well, we examined what types of DAT scales they predict. We divide the scales into 16 clusters by hierarchical clustering based on their $I_{\mathrm{A}}$ sets. In Fig. 6A we display the $I_{\mathrm{A}}$ distribution and geographical composition for each cluster. Middle Eastern, Western, South Asian and East Asian scales appear to have been at one time based on similar mathematics [12 55 58, so it might be expected that they should cluster together $(\mathrm{a}, \mathrm{c}, \mathrm{j}, \mathrm{l})$. There are similarities between some South East Asian and African cultures that utilise equidistant 5 and 7-note scales $(\mathrm{d}, \mathrm{h}, \mathrm{i}, \mathrm{p})$. Clearly there are also some clusters that might be ill-defined (e, k).

The number of scales in each cluster found by the TRANS and FIF models is shown in Fig. 6B. For clarity, we omit the HAR results as they are qualitatively similar to the FIF results, but with less scales predicted (SI Fig $6 \mathrm{~A}$ ). The conclusions drawn about the differences between the TRANS and FIF models are also relevant when comparing the TRANS and HAR models. All models appear to perform well when $I_{\mathrm{A}}$ distributions are uni/bimodal with sharp peaks (a, b, c, i). The
TRANS model performs better if $I_{\mathrm{A}}$ distributions have sharp peaks, regardless of how many peaks there are (a, c, d, i, j, l). The harmonicity models are better adapted to scales from clusters with broad $I_{\mathrm{A}}$ distributions (b, e, f, g, h, m, n). Despite these differences, the majority of scales that are found are common to all models, and while the TRANS and FIF (HAR) cost functions are not correlated in MIN scales (Pearson's $r=0.01$ $(-0.04))$ they are correlated in DAT scales (Pearson's $r=0.41$ (0.12)) (SI Fig 7). Ultimately, of those scales that are predicted, a majority appear to be selected for both harmonicity and transmittability.

\section{A majority of scales were predicted by at least one model}

Between the three models, 407 out of $742(55 \%)$ scales were found. There are several reasons why the other scales were not found: (i) It is impossible for the model to generate them due to having an interval smaller than $I_{\min }$ or deviations from a perfect octave. (ii) It is possible but unlikely for the model to generate them due to having intervals at the tails of the $I_{\mathrm{A}}$ distributions - this is a feature of the random sampling, which is compounded by the use of an $I_{\min }$ constraint. (iii) They are likely to be found, but we generated too few samples to find them all. (iv) They are not likely to be found by any model.

We calculated the breakdown of the reasons why scales were not found (Fig. 6 C). Scales are in category (i) if $I_{\min }<70$ or if the octave deviates from 1200 cents by more than 10 cents 
(155 scales). For all scales we calculate the probability, $P_{\text {MIN }}$, that they are predicted after applying the $I_{\min }$ constraint, and note the value of $P_{\text {MIN }}$ below which only $10 \%$ of scales are not found. Scales with $P_{\text {MIN }}$ lower than this value are in category (ii) (26 scales), or in one of categories (iii) and (iv) otherwise. We calculate for all scales the probability, $P_{\mathrm{ANY}}$, that a scale will be found by any model, and note the value of $P_{\mathrm{ANY}}$ below which only $10 \%$ of scales are not found. Scales with $P_{\mathrm{ANY}}$ higher than this value are in category (iii) (100 scales), otherwise (iv) (54 scales). When we only consider scales that the model can predict (categories ii-iv), we find that the three models are not particularly sensitive to 'theory' or 'measured' scales, nor the region or culture from which they come (SI Fig 6). This implies that these biases are in effect consistently across musical traditions.

\section{Discussion}

Packing arguments account for the success of the harmonicity models

The FIF model manages to reproduce the main features of the interval $\left(I_{\mathrm{A}}\right)$ and scale distributions, simply by specifying inclusion of fifths. This may seem counter-intuitive, but it can be rationalised entirely in terms of packing. Consider first Fig. 4 Since 0 and 1200 cents are fixed points, the easiest way to add fifths is by having notes at $500(1700)$ or 700 cents. Taking into account the circular nature of scales, one can subsequently add notes at 200 (1400) cents or 1000 cents, which will further interact with previously added notes to create more fifths. As a result, there are exclusion zones at either side of 500 and 700 cents. Packing also explains the features of the $I_{\mathrm{A}}$ distributions in Fig. 3 if 200, 500, 700 and 1000 cents are all highly probable, then the $I_{\mathrm{A}}$ distributions ought to contain many 200 cents intervals regardless of $N$.

It is likely that packing is also the main mechanism in the HAR model. The HAR model is affected not only by the harmonic template (Fig. 1B), but also the constraints of fixed $N$ and octave size. The HAR results then arise from the ways of arranging a scale to maximize its average harmonicity score. The fifth is the most important interval in the HAR model, as evidenced by its high score and also the high correlation between the FIF and HAR cost functions (SI Fig 5B). The similarities between the FIF and HAR results (Fig. 34 and SI Fig. 6A) provides additional evidence that the HAR model shares the FIF packing mechanism. Constructing scales from fifths is an old concept 11. The new understanding is that different cultures can be explained in this way even when there is no evidence that they explicitly tuned instruments using fifths (SI Fig. 6).

\section{The devil in music? - The devil is in the detail}

The tritone interval ( $\sim 600$ cents) has been traditionally considered dissonant in Western music - earning it the name diabolus in musica 59 - and is uncommon in classical and folk music 60]. Is it rare because it is unpleasant, or unpleasant because it is rare? Viewing scales as a packing problem reveals an alternative explanation: as $N$ increases, the average $I_{\mathrm{A}}$ size decreases, and it becomes easier to simultaneously pack both tritones and fifths. Analysis of the database shows a linear relationship between $N$ and the frequency of tritone intervals, and this trend is replicated best by the FIF model (SI Fig. 8). Therefore according to this theory, the tritone is rarely used in music simply because it is difficult to simultaneously pack tritones and fifths.

\section{On the universality of harmonic intervals}

The oft-repeated claim that harmonic intervals are universal is based on sparse statistical evidence [59]. Our data shows that harmonicity and prevalence are correlated, and this correlation depends on $N(N=5, r=0.70, p<0.005 ; N=6, r=0.43$, $p>0.05 ; N=7, r=0.65, p<0.005$; SI Fig. 9). Fifths $(3 / 2$, 702 cents) and, due to inversion, fourths $(3 / 2,498$ cents) are the most widespread. However the ratios $7 / 6$ (267 cents) and $7 / 4$ (968 cents) are quite rare despite their relatively high harmonicity, in contrast to $11 / 7$ (783 cents) which is more common yet less harmonic. Thus we report the first clear evidence for prevalence of harmonic intervals, showing that the correlation between prevalence and harmonicity is not straightforward.

Multiple selection pressures affect evolution of scales

All three models, HAR, FIF and TRANS, explain the empirical data significantly better than chance. Thus it is possible that scales are simultaneously optimized for compressibility, maximization of fifths, and maximization of other harmonic intervals. Out of the two harmonicity models, the FIF model better fits the data than the HAR model, but we note that these models are the two extreme cases in how they treat higher order harmonics. A more sophisticated model may have a nuanced approach that accounts for the spectra of natural sounds and how they are processed. There is also a speculative scenario in which harmonic intervals are selected through a different mechanism. The cultures which use small-integer frequency ratios ('theory' scales) overlap with those that had contributed to the development of mathematics 61. Thus the use of mathematics may have contributed to the development of scales 62.

A significant minority of scales are not supported by any model. A view some may consider natural is that perhaps no one mathematical model can predict the diversity of scales. Regarding the theories tested: cultures which produce monophonic music may ignore harmony [19]; cultures which primarily focus on rhythm and dance may not mind imprecision in melodies. Consider the Gamelan pelog scale, variations of which account for over two thirds of cluster p. Pelog scales rarely contain imperfect fifths (SI Fig. 10A). A crude approximation of the pelog scale reduces it to intervals on a 9-TET (9-tone equal temperament) scale 63. This scale is composed of 5 small intervals (average size 133 cents) and 2 large intervals (average size 267 cents). This should be predicted by the TRANS model, but within individual pelog scales the deviations from 9-TET are so large that this rarely happens.
448 449 450 451 452 453 454 455 
The pelog scale is not exceptional in this regard; huge deviations from the average are seen in Thai scales and the Gamelan slendro scale (SI Fig. 10). In this case, some propose that the deviations from some mathematical ideal are not examples of mistuning, but rather a display of artistic intent 64. 66. Tuning as a means of expression is rare in the West, but not unheard of in classical or popular music (see La Monte Young's "The Well-tuned Piano" or King Gizzard and the Wizard Lizard's "Flying Microtonal Banana"). While these theories have performed well, the variability in scales of some cultures hints that although scales are inherently mathematical, mathematics alone lacks the power to fully explain our choices of scales.

\section{Limitations due to assumptions and data}

We report that the FIF model best fits the available data, and we assume that the data is representative of scales used in different musical traditions. However, a different data collection method may significantly alter the results. We replicated the analysis with sub-samples of our database and found that the conclusions are at least robust to resampling (SI Fig 11). Future work may benefit from controlling for historical relationships between musical traditions [4].

Despite evidence that tonal hierarchies are common, they are not a necessary element of scales so we avoid assumptions about tonality [2, 67. We could have assumed that the tonic (the note at 0 cents) is special, and so are intervals made with the tonic. This would explain why in Fig. 4 the 700 cents is more salient than 500 cents. We note that the we could have chosen a different proxy measure of harmonicity 39. However, we think that doing so would not alter the conclusions as the different measures are highly correlated (SI Fig 5A).

\section{Lack of hexatonic scales may be owing to historical} convention

Why do we choose $N$ notes in a scale? - It is generally agreed that there is some trade-off involved. With too few notes melodies lack complexity; with too many notes melodies are difficult to learn. Too few notes results in larger intervals which are more difficult to sing [68; too many notes results in smaller intervals which have lower harmonic similarity [8]. This simple trade-off is at odds with the contrasting ubiquity of 5 and 7 note scales and scarcity of 6 note scales. One suggestion is that 6 note scales are actually prevalent, but classified as variants of 5 and 7 note scales due to convention. Evidence for this can be found in the Essen folk song collection 69.

Chinese music and Western music are conventionally thought to be composed using 5 and 7 note scales respectively, but for both cultures, 6 note scales are the second most prevalent in folk songs (SI Fig. 12). While the following is mere speculation, this work points to a possible route by which a preference for pentatonic and heptatonic scales may have arisen. Given the simplicity of equidistant scales, they are the easiest compressible scales to evolve. For $5,6,7$ and 8 note equidistant scales, the closest notes to fifths are 720 cents, 600 (800) cents, 686 cents, and 750 cents, respectively. Thus, 5 and
7 note scales are the only equidistant scales that can include imperfect fifths. The paucity of recorded hexatonic scales may therefore be due to historical convention driven by evolutionary pressures in early music.

\section{Conclusion}

By constructing a cross-cultural database and using generative stochastic modelling, we quantitatively tested several theories on the origin of scales. Scales tend to include imperfect fifths, and the features of the empirical distributions arise from the most probable ways of packing fifths into a scale of fixed length. Scales also tend to be compressible, which we suggest leads to melodies that are easier to communicate and remember. There is evidence that efficient data compression is a general mechanism humans use for discretizing continuous signals. The effect of compressibility on pitch processing merits further research.

No single theory could explain the diversity exhibited in the database. It is instead likely that scales evolved subject to different selection pressures across cultures. These theories can be further tested by expanding the database, in particular by computationally identifying scales used in ethnographic recordings. Out of two harmonicity-based theories, the one that best fits the empirical data only considers the first three harmonics. This may shed some light on the important, but still developing, understanding of how harmonicity is processed in the brain.

\section{Methods}

The stochastic models

The stochastic models generate adjacent intervals $I_{\mathrm{A}}$ from a uniform distribution, which are then scaled so that they sum to 1200 cents. Some models apply a minimum interval constraint, $I_{\min }$, such that no $I_{\mathrm{A}}$ is smaller than $I_{\min }$. Depending on the model, scales are accepted or rejected according to a probability

$$
P=\min \{1, \exp (-\beta C)\},
$$

where $C$ is a cost function, and we tune the strength of the bias with the parameter $\beta$. We tested many cost functions to check that the results are insensitive to the exact functional form (SI Fig. 13, 14, 15).

For the harmonicity model HAR,

$$
C_{\mathrm{HAR}}=1-\frac{\bar{H}-\bar{H}_{\min }}{\bar{H}_{\max }-\bar{H}_{\min }},
$$

where $\bar{H}_{\min }$ and $\bar{H}_{\max }$ are normalization constants (these constants, obtained via random sampling, are listed in SI Table 4 ), and the average harmonicity score $\bar{H}$ is given by

$$
\bar{H}=[N(N-1)] \sum_{i=0}^{-1} \sum_{j=i+1}^{N-1} H\left(I_{i j}, w\right),
$$

where $H(I)$ is the harmonic similarity score of an interval size $I, w$ is the size of the window in which intervals are considered 
equivalent, and $I_{i j}$ is the interval between note $i$ and note $j$. The index $i=0$ refers to the starting note of the scale and $j$ takes into account the circular nature of scales (if $j>N$ then note $j$ is an octave higher than note $j-N$ ). Note that we do not consider either unison or octave intervals in this score. The harmonic similarity score $H$ of an interval is calculated from its frequency ratio expressed as a fraction.

$$
H(I)=\frac{x+y-1}{x y} \times 100,
$$

where $x$ is the numerator and $y$ is the denominator of the fraction. The harmonic similarity template is produced by creating a grid of windows of maximum size $w$ centred about the intervals that have the largest $H$ values. An interval expressed in cents is allocated to the window with the highest $H$ value that is within $w / 2$ cents.

For the imperfect fifths model FIF,

$$
C_{\mathrm{FIF}}=(1+\bar{F})^{-1}
$$

where a constant is added to prevent division by zero in the case of scales with no imperfect fifths, and the fraction of fifths $\bar{F}$ is

$$
\bar{F}=[N(N-1)] \sum_{i=0}^{-1} \sum_{j=i+1}^{N-1} F\left(I_{i j}, w\right),
$$

where $F(I, w)=1$ if $|I-702| \leq w / 2$ and 0 otherwise.

For the transmittable scales model TRANS,

$C_{\text {TRANS }}=\min _{\frac{1}{2} \min \left\{I_{\mathrm{A}}\right\} \leq \gamma \leq \frac{3}{2} \min \left\{I_{\mathrm{A}}\right\}}\left\{\sum_{i=1}^{N}\left|\left\lceil\frac{I_{A i}}{\gamma}\right\rceil-\frac{I_{A i}}{\gamma}\right|^{n}\right\} / N$,

where $I_{A i}$ is the $i^{\text {th }}$ adjacent interval in a scale, and $n$ is parameter that controls how deviations from the template are considered in the model (see main text). The parameter $\gamma$ is the common denominator of the compressible template; it is constrained so that it is never less than half of the smallest adjacent interval.

\section{Classification of scales as similar}

The fraction of DAT scales $f_{\mathrm{D}}$ for each model was calculated by checking if model scales are similar to DAT scales. Two scales A and B are similar if

$$
\forall i \in\{1 . . N\},\left|\alpha_{i}^{A}-\alpha_{i}^{B}\right| \leq e,
$$

where $\alpha_{i}^{A}$ is $i^{t h}$ note in scale $A$ and the tolerance is $e=10$.

\section{Probability of predicting scales}

$P_{\text {MIN }}$, the probability of a scale A being predicted by the MIN model, is calculated as the sum of the probabilities of each scale $B$ that can be labelled similar to, or the same as, scale $A$. We use a tolerance of $e=10$, we keep the length of the scale constant, and we consider probabilities at an integer resolution, so there are $21^{N-1}$ similar scales.

$$
P_{\mathrm{MIN}}=\sum^{21^{N-1}} \prod_{i=1}^{N} p\left(I_{\mathrm{A} i}^{B}\right)
$$

where $p$ is the probability of an interval being picked by the MIN model, and $I_{\mathrm{A} i}^{B}$ is the $i^{\text {th }}$ adjacent interval in scale B.

$P_{\mathrm{ANY}}$, the probability that any of the TRANS, HAR or FIF models finds a scale is given by

$$
P_{\mathrm{ANY}}=P_{\mathrm{MIN}} \sum P(\beta, C),
$$

where $P$ is summed over the three models using the parameters listed in SI Table 4.

\section{Clustering criterion}

To group DAT scales we used hierarchical clustering based on distances between adjacent intervals. The distance, $d_{A B}$, from scale $A$ to scale $B$ is asymmetric, and is calculated as the sum of the shortest distances from every $I_{\mathrm{A}}$ in $A$ to any $I_{\mathrm{A}}$ in $\mathrm{B}$,

$$
d_{A B}=\sum_{i=1}^{N} \min _{j}\left\{I_{\mathrm{A} i}^{A}-I_{\mathrm{A} j}^{B}\right\},
$$

where $i$ and $j$ are the indices of adjacent intervals in scales $A$ and $B$ respectively. For clustering, we use the symmetric distance $d_{C}=\left(d_{A B} d_{B A}\right)^{1 / 2}$. We used Ward's minimum variance method to agglomerate clusters using the SciPy package for Python 70 .

\section{Statistical analysis}

We tested whether the empirical $I_{\mathrm{A}}$ and note distributions are better approximated by a theoretical model (FIF,HAR,TRANS), compared to the RAN, and the MIN model, which are effectively the null distributions. We chose to smooth the $I_{\mathrm{A}}$ distributions in Fig. 3 mainly due to one assumption. Given that (with the exception of modern fixed pitch instruments) intervals are not produced as exact frequency ratios, the $I_{\mathrm{A}}$ distributions should be smooth. We consider that the sharp peaks (e.g., at 100 and 200 cents are a result of theoretical values for interval sizes and are not representative of how intervals are produced in reality. To minimize artefacts from this smoothing we used a non-parametric kernel density estimation method, implemented in the Statsmodels package for Python 71. The kernel is Gaussian and Silverman's rule is used to estimate the bandwidth. We compared goodness-of-fit between the empirical and theoretical distributions using the Jensen-Shannon divergence (we get the arithmetic mean across $N$, weighted by sample size). We verified that the smoothing did not influence the results by using a two sample Cramér-von Mises test (SI Table 5). Due to the high dimensionality of the system we needed to generate large samples $\left(S=10^{4}\right)$ for each model. As a result, $p$ values tend to be astronomically low $(p \ll 0.01$ ), so we instead calculated $95 \%$ confidence intervals by bootstrapping (1000 resamples from 
the DAT sample). Applying kernel density estimation to the note distributions (Fig. 4 ) resulted in a loss of detail via oversmoothing. Hence, we only show histograms for these distributions and calculate the Jensen-Shannon divergence using the histograms. The conclusions do not depend on the histogram bin size. To test whether we have sufficient empirical data we replicate the analysis with three types of sub-sampling: bootstrapping with smaller sample sizes; only the 'theory' scales; and only the 'measured' scales (SI Fig 11). We find that the conclusions are robust to resampling.

\section{Data and Code Availability}

All data used in the figures and Supplementary Information are available, along with simulation and analysis code are accessible at https://github.com/jomimc/imperfect_fifths. The scales database is included as Supplementary Material.

\section{Acknowledgements}

J.M. acknowledges helpful discussions with Patrick Savage. This work was supported by the taxpayers of South Korea through the Institute for Basic Science, Project Code IBSR020-D1.

\section{Competing Interests}

The authors declare that they have no competing financial interests.

\section{Correspondence}

Correspondence and requests for materials should be addressed to J.M. (email: jmmcbride@protonmail.com) and T.T. (tsvitlusty@gmail.com).

\section{Author Contributions}

J.M. and T.T. designed research; J.M. performed research; J.M. analyzed data; J.M. and T.T. wrote the paper.

\section{References}

[1] C. Darwin. The Descent of Man and Selection in Relation to Sex, volume 1. D. Appleton, 1896.

[2] J. McDermott and M. Hauser. The origins of music: Innateness, uniqueness, and evolution. Music Percept., 23 (1):29-59, 2005. doi: $10.1525 / \mathrm{mp} .2005 .23 .1 .29$.

[3] W. T. Fitch. The biology and evolution of music: A comparative perspective. Cognition, 100(1):173 - 215, 2006. doi: https://doi.org/10.1016/j.cognition.2005.11.009.

[4] P. Ball. The Music Instinct: How Music Works and Why We Can't Do Without It. Random House, 2010.

[5] N. L. Wallin, B. Merker, and S. Brown. The Origins of Music. MIT press, 2001.
[6] B. Nettl. The Study of Ethnomusicology: Thirty-One Issues and Concepts. University of Illinois Press, 2010.

[7] Steven Brown and Joseph Jordania. Universals in the worlds musics. Psychol. Music, 41(2):229-248, 2013. doi: $10.1177 / 0305735611425896$.

[8] K. Z. Gill and D. Purves. A biological rationale for musical scales. Plos One, 4(12):1-9, 2009. doi: 10.1371/journal. pone.0008144.

[9] D. A. Schwartz, C. Q. Howe, and D. Purves. The statistical structure of human speech sounds predicts musical universals. J. Neurosci., 23(18):7160-7168, 2003. doi: 10.1523/JNEUROSCI.23-18-07160.2003.

[10] Laetitia Bruckert, Patricia Bestelmeyer, Marianne Latinus, Julien Rouger, Ian Charest, Guillaume A. Rousselet, Hideki Kawahara, and Pascal Belin. Vocal attractiveness increases by averaging. Curr. Biol., 20(2):116 - 120, 2010. doi: https://doi.org/10.1016/j.cub.2009.11.034.

[11] M. L. West. The babylonian musical notation and the hurrian melodic texts. Music Lett., 75(2):161-179, 1994. doi: $10.2307 / 737674$.

[12] H. Rechberger. Scales and Modes Around the World: The Complete Guide to the Scales and Modes of the World. Fennica Gehrman Ltd., 2018.

[13] J. Kuroyanagi, S. Sato, M. J. Ho, G. Chiba, J. Six, P. Pfordresher, A. Tierney, S. Fujii, and P. Savage. Automatic comparison of human music, speech, and bird song suggests uniqueness of human scales. In Proceedings of the Folk Music Analysis (FMA 2019), 2019.

[14] E. M. Burns. Intervals, scales, and tuning. In Diana Deutsch, editor, The Psychology of Music (Second Edition), pages 215 - 264. Academic Press, San Diego, second edition edition, 1999. doi: https://doi.org/10.1016/ B978-012213564-4/50008-1.

[15] A. D. Patel. Music, Language, and the Brain. Oxford university press, 2010.

[16] Josh H. McDermott, Andriana J. Lehr, and Andrew J. Oxenham. Individual differences reveal the basis of consonance. Curr. Biol., 20(11):1035 - 1041, 2010. doi https://doi.org/10.1016/j.cub.2010.04.019.

[17] M. Cousineau, J. H. McDermott, and I. Peretz. The basis of musical consonance as revealed by congenital amusia P. Natl. Acad. Sci. Usa., 109(48):19858-19863, 2012. doi: 10.1073/pnas.1207989109.

[18] D. L. Bowling and D. Purves. A biological rationale for musical consonance. P. Natl. Acad. Sci. Usa., 112(36): 11155-11160, 2015. doi: 10.1073/pnas.1505768112. 
[19] J. H. McDermott, A. F. Schultz, E. A. Undurraga, and R. A. Godoy. Indifference to dissonance in native amazonians reveals cultural variation in music perception. Nature, 535:547 EP -, 2016. doi: 10.1038/nature18635.

[20] Nori Jacoby, Eduardo A. Undurraga, Malinda J. McPherson, Joaqun Valds, Toms Ossandn, and Josh H. McDermott. Universal and non-universal features of musical pitch perception revealed by singing. Curr. Biol., 2019. doi: 10.1016/j.cub.2019.08.020.

[21] P. E. Savage. The Need for Global Studies. Oxford University Press, New York, 2019.

[22] Peter Q. Pfordresher and Steven Brown. Vocal mistuning reveals the origin of musical scales. Eur. J. Cogn. Psychol., 29(1):35-52, 2017. doi: 10.1080/20445911.2015. 1132024.

[23] S. Sato, J. Six, P. Pfordresher, S. Fujii, and P. Savage. Automatic comparison of global childrens and adult songs supports a sensorimotor hypothesis for the origin of musical scales. In 9th Folk Music Analysis Conference, 2019. doi: https://doi.org/10.31234/osf.io/kt7py.

[24] J. A. Siegel and W. Siegel. Categorical perception of tonal intervals: Musicians cant tell sharp from flat. Percept. Psychophys., 21(5):399-407, 1977. doi: 10.1037/ h0094008.

[25] E. M. Burns and W. D. Ward. Categorical perceptionphenomenon or epiphenomenon: Evidence from experiments in the perception of melodic musical intervals. J. Acoust. Soc. Am., 63(2):456-468, 1978. doi: 10.1121/1.381737.

[26] M. P. Lynch, R. E. Eilers, D. K. Oller, R. C. Urbano, and P. Wilson. Influences of acculturation and musical sophistication on perception of musical interval patterns. J. Exp. Psychol. Human., 17(4):967, 1991. doi: 10.1037/ 0096-1523.17.4.967.

[27] J. H. McDermott, M. V. Keebler, C. Micheyl, and A. J. Oxenham. Musical intervals and relative pitch: Frequency resolution, not interval resolution, is special. $J$. Acoust. Soc. Am., 128(4):1943-1951, 2010. doi: 10.1121/ 1.3478785 .

[28] J. Serra, G. K. Koduri, M. Miron, and X. Serra. Assessing the tuning of sung indian classical music. In $I S M I R$, pages 157-162, 2011.

[29] J. Devaney, M. I. Mandel, D. P. W. Ellis, and I. Fujinaga. Automatically extracting performance data from recordings of trained singers. PsychoMusicology, 21(1-2):108, 2011. doi: $10.1037 / \mathrm{h} 0094008$.

[30] F. A. Russo and W. F. Thompson. An interval size illusion: The influence of timbre on the perceived size of melodic intervals. Percept. Psychophys., 67(4):559-568, 2005. doi: 10.3758/BF03193514.
[31] F. A. Russo and W. F. Thompson. The subjective size of melodic intervals over a two-octave range. Psychon. $B$. Rev., 12(6):1068-1075, 2005. doi: 10.3758/BF03206445.

[32] C. Aruffo, R. L. Goldstone, and D. J. D. Earn. Absolute judgment of musical interval width. Music Percept., 32 (2):186-200, 2014. doi: 10.1525/mp.2014.32.2.186.

[33] John M. Geringer, Rebecca B. MacLeod, and Justine K. Sasanfar. In tune or out of tune: Are different instruments and voice heard differently? J. Res. Music Educ., 63(1): 89-101, 2015. doi: 10.1177/0022429415572025.

[34] P. Larrouy-Maestri, P. M. C. Harrison, and D. Müllensiefen. The mistuning perception test: A new measurement instrument. Behav. Res. Methods, 51 (2):663-675, 2019. doi: 10.3758/s13428-019-01225-1.

[35] T. Cowen. Creative Destruction: How Globalization Is Changing the World's Cultures. Princeton University Press, 2009.

[36] J. Six and O. Cornelis. Tarsos: A platform to explore pitch scales in non-western and western music. In 12th International Society for Music Information Retrieval Conference (ISMIR-2011), pages 169-174, 2011.

[37] R. Plomp. The ear as a frequency analyzer. J. Acoust. Soc. Am., 36(9):1628-1636, 1964. doi: 10.1121/1.1919256.

[38] C. J. Plack and A. J. Oxenham. The psychophysics of pitch. In Pitch, pages 7-55. Springer, 2005.

[39] P. Harrison and M. Pearce. Simultaneous consonance in music perception and composition. Psychol. Rev., 2019.

[40] W. M. Hartmann. On the origin of the enlarged melodic octave. J. Acoust. Soc. Am., 93(6):3400-3409, 1993. doi: $10.1121 / 1.405695$

[41] F. Loosen. The effect of musical experience on the conception of accurate tuning. Music Percept., 12(3):291-306, 1995. doi: $10.2307 / 40286185$.

[42] Edward C. Carterette and Roger A. Kendall. On the tuning and stretched octave of javanese gamelans. Leonardo Music J., 4:59-68, 1994. doi: 10.2307/1513182.

[43] K. P. Wachsmann. An equal-stepped tuning in a ganda harp. Nature, 165(4184):40-41, 1950. doi: 10.1038/ 165040a0.

[44] W. Van Zanten. The equidistant heptatonic scale of the asena in malawi. Afr. Music, 6(1):107-125, 1980. doi: 10.21504/amj.v6i1.1099.

[45] L. E. McNeil and S. Mitran. Vibrational frequencies and tuning of the african mbira. J. Acoust. Soc. Am., 123(2): 1169-1178, 2008. doi: 10.1121/1.2828063. 
[46] Barry Ross and Sarah Knight. Reports of equitonic scale systems in african musical traditions and their implications for cognitive models of pitch organization. $\mathrm{Mu}$ sic. Sci., page 1029864917736105, 2017. doi: 10.1177/ 1029864917736105.

[47] P. E. Savage, S. Brown, E. Sakai, and T. E. Currie. Statistical universals reveal the structures and functions of human music. P. Natl. Acad. Sci. Usa., 112(29):89878992, 2015. doi: 10.1073/pnas.1414495112.

[48] M. S. Lewicki. Efficient coding of natural sounds. Nat. Neurosci., 5(4):356-363, 2002. doi: 10.1038/nn831.

[49] Peter Desain and Henkjan Honing. The formation of rhythmic categories and metric priming. Perception, 32 (3):341-365, 2003. doi: 10.1068/p3370.

[50] A. Ravignani, T. Delgado, and S. Kirby. Musical evolution in the lab exhibits rhythmic universals. Nature Human Behaviour, 1:0007 EP -, 2016. doi: 10.1038/ s41562-016-0007.

[51] Nori Jacoby and Josh H. McDermott. Integer ratio priors on musical rhythm revealed cross-culturally by iterated reproduction. Curr. Biol., 27(3):359 - 370, 2017. doi: https://doi.org/10.1016/j.cub.2016.12.031.

[52] C. E. Stilp and K. R. Kluender. Cochlea-scaled entropy, not consonants, vowels, or time, best predicts speech intelligibility. P. Natl. Acad. Sci. Usa., 107(27):12387-12392, 2010. doi: 10.1073/pnas.0913625107.

[53] T. Regier, P. Kay, and N. Khetarpal. Color naming reflects optimal partitions of color space. P. Natl. Acad. Sci. Usa., 104(4):1436-1441, 2007. doi: 10.1073/pnas. 0610341104.

[54] Roland Baddeley and David Attewell. The relationship between language and the environment: Information theory shows why we have only three lightness terms. Psychol. Sci., 20(9):1100-1107, 2009. doi: 10.1111/j. 1467-9280.2009.02412.x.

[55] E. te Nijenhuis. Dattilam: A Compendium of Ancient Indian Music, volume 11. Brill Archive, 1970.

[56] K. L. Signell. Makam: Modal Practice in Turkish Art Music, volume 4. Da Capo Pr, 1977.

[57] H. Farhat. The Dastgah Concept in Persian Music. Cambridge University Press, 2004.

[58] I. Katz. Henry George Farmer and the First International Congress of Arab Music (Cairo 1932). Brill, 2015.

[59] S. E. Trehub. Human processing predispositions and musical universals. In Henkjan Honing, editor, The origins of musicality. The MIT Press, 2018.
[60] P. G. Vos and J. M. Troost. Ascending and descending melodic intervals: Statistical findings and their perceptual relevance. Music Percept., 6(4):383-396, 1989. doi: 10. $2307 / 40285439$.

[61] M. K. J. Goodman. An Introduction to the Early Development of Mathematics. John Wiley \& Sons, 2016.

[62] Richard L. Crocker. Pythagorean mathematics and music. The Journal of Aesthetics and Art Criticism, 22(2):189198, 1963. doi: $10.2307 / 427754$.

[63] Jay Rahn. Javanese plog tunings reconsidered. Yearb. Int. Folk Music Council, 10:69-82, 1978. doi: 10.2307/767348.

[64] Roger Vetter. A retrospect on a century of gamelan tone measurements. Ethnomusicology, 33(2):217-227, 1989. doi: $10.2307 / 924396$.

[65] S. Marcus. The interface between theory and practice: Intonation in arab music. Asian Music, 24(2):39-58, 1993. doi: $10.2307 / 834466$.

[66] J. Garzoli. The myth of equidistance in thai tuning. Anal Approaches Music, 4(2):1-29, 2015.

[67] E. J. Kessler, C. Hansen, and R. N. Shepard. Tonal schemata in the perception of music in bali and in the west. Music Percept., 2(2):131-165, 1984. doi: 10.2307/ 40285289 .

[68] J. Sundberg, I. Titze, and R. Scherer. Phonatory control in male singing: A study of the effects of subglottal pressure, fundamental frequency, and mode of phonation on the voice source. J. Voice, $7(1): 15-29,1993$. doi: https://doi.org/10.1016/S0892-1997(05)80108-0.

[69] H. Schaffrath. The essen folksong collection, 1995.

[70] Eric Jones, Travis Oliphant, Pearu Peterson, et al. Scipy: Open source scientific tools for Python, 2001-.

[71] S. Seabold and J. Perktold. Statsmodels: Econometric and statistical modeling with python. In 9th Python in Science Conference, 2010. 


\title{
Supporting Information for Cross-cultural data shows musical scales evolved to maximise imperfect fifths
}

\author{
John M. McBride ${ }^{1, *}$ and Tsvi Tlusty ${ }^{1,2, *}$ \\ ${ }^{1}$ Center for Soft and Living Matter, Institute for Basic Science, Ulsan 44919, South Korea \\ ${ }^{2}$ Department of Physics, Ulsan National Institute of Science and Technology, Ulsan 44919, South Korea \\ *jmmcbride@protonmail.com, tsvitlusty@gmail.com
}

June 1, 2020

\section{Prominent intervals in the harmonic series}

We estimate the prominence of intervals that are made between harmonics in a single harmonic series by weighted counting. We count the intervals made between the first $n_{1}$ harmonics and the first $n_{2}$ harmonics, with each interval combination only counted once. We consider two cases: $n_{1}=1$ and $n_{2}=10 ; n_{1}=10$ and $n_{2}=10$. In the first case, only the intervals made with respect to the fundamental are counted. In the second case, all possible intervals within a harmonic series are counted, up to a maximum harmonic number 10 . We weight the intervals with $\lambda=(1-a)^{i-1}(1-a)^{j-1}$, where $a$ is the attenuation rate, such that the weights decay with harmonic number following a power law. We find for both cases that as the attenuation rate increases the relative counts of intervals diverge (Table 1). E.g., for the second case with $a=0.3$, the ratios between the weighted counts for unison, octave, fifth and major third are approximately $4: 4: 2: 1$; while with $a=0.6$ they are $25: 5: 2: 1$.

\section{Scales database and inclusion criteria}

Scales are obtained from three types of sources. Scales with fixed theoretical interval sizes (labelled 'theory' scales in the main text) are obtained from books 1, 2]. A separate set of scales (labelled 'measured' scales) come from two types of sources: scales which are inferred from measurements of instrument tunings 33 33; scales which are inferred from analysis of recorded musical performances 34 35. A full breakdown is provided in Table 2

All of the 'theory' scales are included at least once in the database, and in many cases more than once if is known that it was used in multiple tuning systems (Table 3. In addition, scales were included in the database according to the following criteria:

- Scales must be reported in sources as intervals in cents or as frequency ratios of notes.

- Only scales with at least 4 and at most 9 notes are included.

- If a scale has notes beyond the octave these notes are excluded.

- Scales within a single culture that are identical within 1 cents tolerance are only included once (i.e. key changes are not considered).

- When the source omits information about the tonic, the tonic is taken as the first note for which a consecutive series of intervals add up to within 50 cents of an octave. Those intervals are only used in one scale (i.e. 'modes' are not inferred from 'measured' scales)

- A number of sources lack the exact value of the final interval 17, 29, 35. If there is evidence that the scale includes the octave, then a final interval is appended so that the scale ends on the octave.

- Scales are excluded if there are significant inconsistencies or errors in the reporting.

\section{Vocal mistuning theory predicts a minimum interval size}

The vocal mistuning theory states that both singing and pitch perception are subject to errors, and hence scales evolved so that they were easy to sing and hear [36. It is often assumed that error distributions are likely to be Gaussian 37-40. By modelling this phenomenon as a transmission problem we assess the effect of interval size on errors in transmission. That is, given Gaussian errors on both interval production and perception, what is the probability that a sung interval will be perceived as it is intended?

Table 1: The most prominent harmonics present in a single harmonic series of length $n_{2}=10$, as calculated by weighted counting. $n_{1}=1$ corresponds to only counting intervals made with respect to the tonic, while $n_{1}=10$ corresponds to counting all possible intervals. $a$ is the attenuation rate: $a=0$ means that all harmonics have equal weights; $a=1$ means that only the first harmonic is counted.

\begin{tabular}{|c|c|c|c|c|c|c|c|c|c|c|c|c|}
\hline \multirow{3}{*}{$\begin{array}{l}n_{1} \\
a\end{array}$} & \multicolumn{6}{|c|}{1} & \multicolumn{6}{|c|}{10} \\
\hline & \multicolumn{2}{|c|}{0} & \multicolumn{2}{|c|}{0.3} & \multicolumn{2}{|c|}{0.6} & \multicolumn{2}{|c|}{0} & \multicolumn{2}{|c|}{0.3} & \multicolumn{2}{|c|}{0.6} \\
\hline & ratio & count & ratio & count & ratio & count & ratio & count & ratio & count & ratio & count \\
\hline & $2 / 1$ & 3.0 & $2 / 1$ & 1.0 & $1 / 1$ & 1.0 & $1 / 1$ & 10.0 & $1 / 1$ & 2.0 & $1 / 1$ & 1.0 \\
\hline & $5 / 4$ & 2.0 & $1 / 1$ & 1.0 & $2 / 1$ & 0.5 & $2 / 1$ & 8.0 & $2 / 1$ & 2.0 & $2 / 1$ & 0.5 \\
\hline & $3 / 2$ & 2.0 & $3 / 2$ & 0.7 & $3 / 2$ & 0.2 & $5 / 4$ & 7.0 & $3 / 2$ & 1.0 & $3 / 2$ & 0.2 \\
\hline & $7 / 4$ & 1.0 & $5 / 4$ & 0.3 & $5 / 4$ & 0.03 & $3 / 2$ & 7.0 & $5 / 4$ & 0.6 & $5 / 4$ & 0.04 \\
\hline & $1 / 1$ & 1.0 & $7 / 4$ & 0.1 & $7 / 4$ & 0.004 & $9 / 8$ & 4.0 & $7 / 4$ & 0.2 & $4 / 3$ & 0.01 \\
\hline & $9 / 8$ & 1.0 & $9 / 8$ & 0.06 & $9 / 8$ & 0.0007 & $7 / 4$ & 3.0 & $4 / 3$ & 0.2 & $7 / 4$ & 0.006 \\
\hline & & & & & & & $4 / 3$ & 3.0 & $5 / 3$ & 0.1 & $5 / 3$ & 0.004 \\
\hline & & & & & & & $5 / 3$ & 3.0 & $9 / 8$ & 0.1 & $9 / 8$ & 0.001 \\
\hline & & & & & & & $7 / 6$ & 2.0 & $7 / 6$ & 0.08 & $7 / 6$ & 0.0007 \\
\hline & & & & & & & $8 / 5$ & 1.0 & $6 / 5$ & 0.04 & $6 / 5$ & 0.0003 \\
\hline
\end{tabular}


Table 2: The number of scales of each type from each source. Theory scales have exact frequency ratios specified for each interval. Instrument scales are inferred from measurements of instrument tunings. Recording scales are inferred computationally from recordings.

\begin{tabular}{|c|c|c|c|c|}
\hline Reference & Theory & Instrument & Recording & Total \\
\hline 2 & 176 & 67 & 0 & 243 \\
\hline 1 & 228 & 0 & 0 & 228 \\
\hline 7 & 0 & 51 & 0 & 51 \\
\hline 11 & 0 & 46 & 0 & 46 \\
\hline 35 & 0 & 0 & 28 & 28 \\
\hline 14 & 21 & 0 & 0 & 21 \\
\hline 30] (raw data obtained via personal communication) & 0 & 17 & 0 & 17 \\
\hline 25 & 0 & 12 & 0 & 12 \\
\hline 27 & 0 & 9 & 0 & 9 \\
\hline 12 & 0 & 7 & 0 & 7 \\
\hline 18 & 0 & 7 & 0 & 7 \\
\hline 9 & 0 & 6 & 0 & 6 \\
\hline 4 & 0 & 6 & 0 & 6 \\
\hline 10 & 0 & 5 & 0 & 5 \\
\hline 33 & 0 & 5 & 0 & 5 \\
\hline 19 & 0 & 5 & 0 & 5 \\
\hline 24 & 0 & 4 & 0 & 4 \\
\hline 34 & 0 & 0 & 4 & 4 \\
\hline 28 & 0 & 4 & 0 & 4 \\
\hline 31 & 0 & 4 & 0 & 4 \\
\hline 22 & 0 & 4 & 0 & 4 \\
\hline 13 & 0 & 4 & 0 & 4 \\
\hline 15 & 0 & 4 & 0 & 4 \\
\hline 17 & 0 & 3 & 0 & 3 \\
\hline 5 & 0 & 3 & 0 & 3 \\
\hline 21 & 0 & 2 & 0 & 2 \\
\hline 29 & 0 & 2 & 0 & 2 \\
\hline 16 & 0 & 2 & 0 & 2 \\
\hline 8 & 0 & 2 & 0 & 2 \\
\hline 20 & 0 & 2 & 0 & 2 \\
\hline 3 & 0 & 2 & 0 & 2 \\
\hline 6 & 0 & 2 & 0 & 2 \\
\hline 32 & 0 & 1 & 0 & 1 \\
\hline 23 & 0 & 1 & 0 & 1 \\
\hline
\end{tabular}

\begin{tabular}{|c|c|c|c|c|c|c|c|c|}
\hline Culture & $\begin{array}{l}\text { 3: The } \\
\text { 12-tet }\end{array}$ & $\begin{array}{l}\text { tunings } \\
24 \text {-tet }\end{array}$ & $\begin{array}{l}\text { used for } \\
53 \text {-tet }\end{array}$ & $\begin{array}{l}\text { heory' scales dep } \\
\text { Just Intonation }\end{array}$ & $\begin{array}{l}\text { Ping on cultu } \\
\text { Pythagorean }\end{array}$ & Persian & Turkish & Shi-er-lu \\
\hline Western Classical (years 1700+) & $\mathrm{X}$ & & & & & & & \\
\hline Jazz & $\mathrm{X}$ & & & & & & & \\
\hline Diatonic modes & $\mathrm{X}$ & & & $\mathrm{X}$ & $\mathrm{X}$ & & & \\
\hline Greek Folk & $\mathrm{X}$ & & & $\mathrm{X}$ & & & & \\
\hline Jewish & $\mathrm{X}$ & & & $\mathrm{X}$ & & & & \\
\hline Japanese & $\mathrm{X}$ & & & & $\mathrm{X}$ & & & \\
\hline Chinese & $\mathrm{X}$ & & & & & & & $\mathrm{X}$ \\
\hline Hindustani & $\mathrm{X}$ & & & $\mathrm{X}$ & & & & \\
\hline Carnatic & & & & $\mathrm{X}$ & & & & \\
\hline Arabian & & $\mathrm{X}$ & $\mathrm{X}$ & & & & & \\
\hline Persian & & & & & & $\mathrm{X}$ & & \\
\hline Turkish & & & $\mathrm{X}$ & & & & $\mathrm{X}$ & \\
\hline
\end{tabular}


A

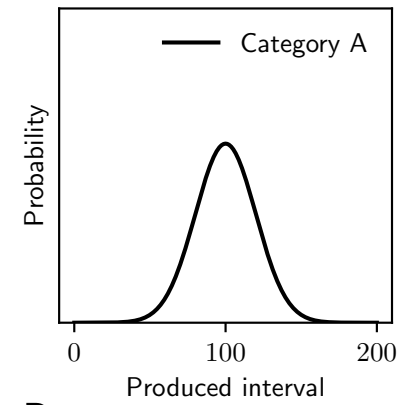

B

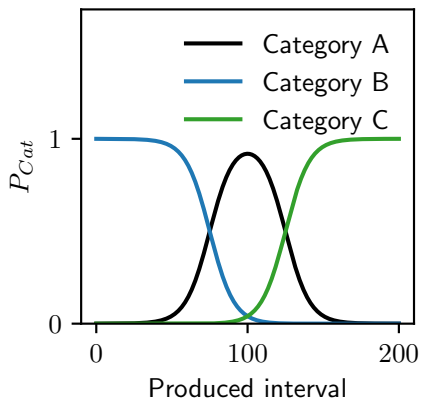

C

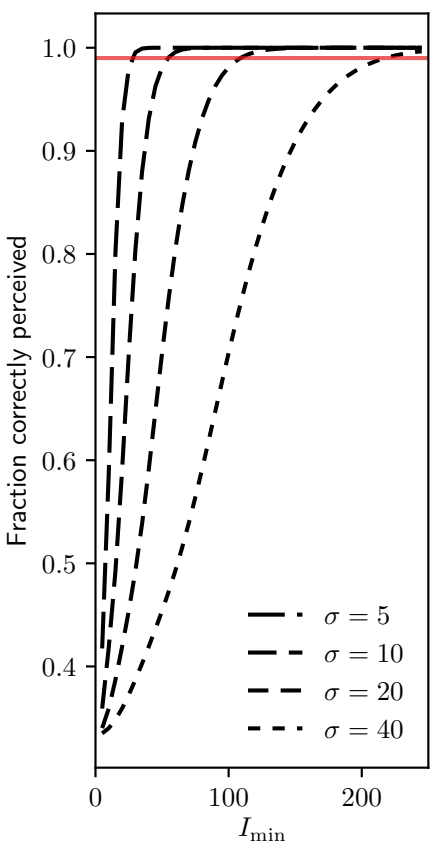

$\mathrm{D}$

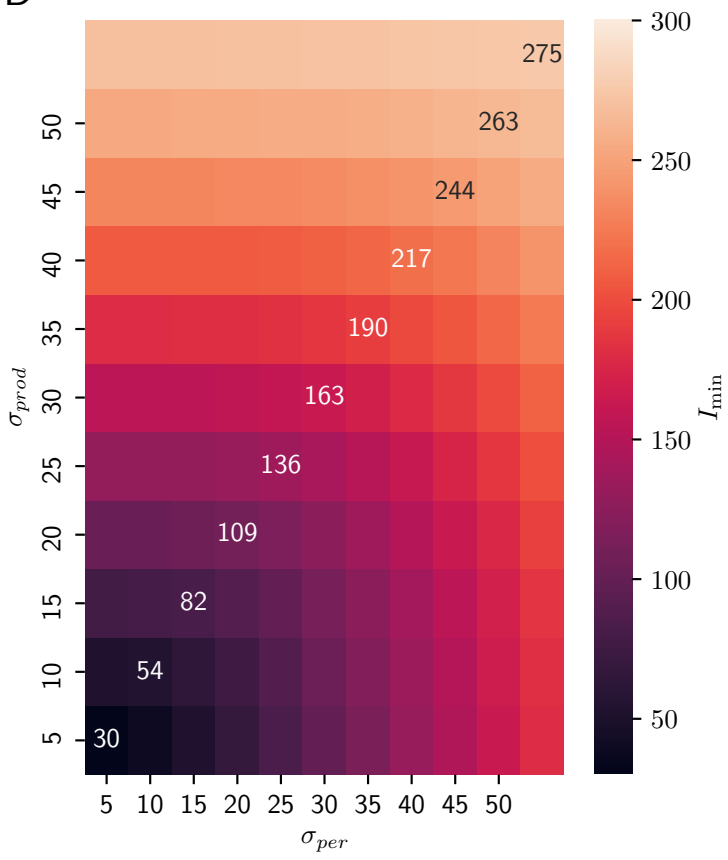

Figure 1: A: Probability distribution of a sung interval which is intended to be 100 cents; $\mu=100$ cents and $\sigma=20$ cents. B: Probability that a sung interval will be heard as a category A, B or C; $\mu=[50,100,150]$ and $\sigma=20$. C: The fraction of intervals that are perceived as intended as a function of the smallest separation between categories, $I_{\text {min }}$; the red line indicates the point at which $99 \%$ of intervals are correctly perceived; $\sigma=\sigma_{\text {prod }}=\sigma_{\text {per }}$. D: The minimum interval size $I_{\text {min }}$ for which $99 \%$ of intervals are perceived correctly, as a function of $\sigma_{\text {per }}$ and $\sigma_{\text {prod }}$.

We vary the standard deviation of the size of the produced interval, $\sigma_{\text {prod }}$, and the standard deviation of the probability that an interval $I$ will be perceived as a specific category, $\sigma_{\text {per }}$. The means of interval categories are separated by a distance $I_{\text {min }}$. The probability, $P_{\text {Cat }}$, that an interval $I$ will be perceived as a category A is

$$
P_{\text {Cat }}=\exp \left(-\frac{\left(I-\mu_{\mathrm{A}}\right)^{2}}{2 \sigma_{\text {per }}^{2}}\right)\left[\sum_{X} \exp \left(-\frac{\left(I-\mu_{\mathrm{X}}\right)^{2}}{2 \sigma_{\text {per }}^{2}}\right)\right]^{-1},
$$

where $\mu_{\mathrm{X}}$ is the mean of any category $\mathrm{X}$. Thus we can calculate the fraction of correctly perceived intervals as a function of the distance between interval categories $I_{\min }$ (Fig. $1 \mathrm{C}$ ). We then define an acceptable minimum interval size as that which corresponds to $99 \%$ of intervals being correctly identified. This allows us to determine a minimum interval size which depends on $\sigma_{\text {prod }}$ and $\sigma_{\text {per }}$ (Fig. 1P).

While we lack systematic studies of the range of errors of humans' ability to sing or identify intervals accurately, a few studies provide information which allows one to speculate. Studies on barbershop quartets and Indian classical music respectively report $\sigma_{\text {prod }} \approx 4-17$ cents and $\sigma_{\text {prod }} \approx 9-15$ cents 39,41 . A study including untrained, amateur and professionals as subjects reported errors in sung intervals ranging from approximately 10 to 200 cents [36]. Studies on interval perception show that interval discrimination has a similar range of errors 38, 42. One study reported just-noticeable differences ranging from 4 cents (professional) to $\sim 40$ cents (untrained) 43 . Another study found that musicians who can correctly identify Western-classical interval categories accurate to 100 cents generally did not discern inaccuracies of 20 cents [37. From this we suggest that expert musicians can be thought of having $\sigma_{\mathrm{prod}} \approx \sigma_{\mathrm{per}} \approx 10$ which results in a lower limit of $I_{\mathrm{min}}=54$. If minimising errors in transmission is important, it would be inadvisable to use scales with intervals close to the $I_{\text {min }}$ of a trained expert. The predictions of this approach accord with the empirical observation that in reality such small intervals are rarely used. Arabic music is one of the few examples of a culture using 50 cents intervals, and they are considered ornamental by many. For the untrained person, it is likely preferable that intervals should be considerably larger.

\section{Vocal mistuning theory predicts equidistant scales}

For any convex, monotonically decreasing cost function $C=f(I)$, where $\sum_{i}^{N} I_{i}=1200, \sum_{i}^{N} C_{i}$ will be minimized by setting each $I_{i}=1200 / N$. For a concave function, a minimum is found as one interval $j$ tends to the limit $I_{j} \rightarrow 1200$ while the other intervals $k$ tend to zero $\sum^{N-1} I_{k} \rightarrow 0$. The marginal case is that of a linear function, which does not lead to a bias, implying that the theory does not affect the choice of scales.

The vocal mistuning theory states that as interval size increases the probability of miscommunication decreases 36 , but we do not know the form of the corresponding cost function. If we want to improve accuracy in transmission, are we more concerned with having fewer small intervals or having more large intervals? If it is the former, then the cost function is convex, while if it is the latter then the function is concave. Fig. $1 \mathrm{C}$ shows that as $I_{\min }$ increases the accuracy saturates. This means that the cost function cannot diverge as $I$ increases, so it must be a convex function. Hence, the vocal mistuning theory predicts equidistant scales.

Equidistant scales are typically considered rare 44, and non-equidistant scales are professed to have advantages (e.g., tonality) over equidistant scales 45, 48. However many scales are almost equidistant 3, 11, 27, 49, and there may be fewer equidistant scales simply because the base probability is low due to there being fewer ways to construct an equidistant scale. 
Table 4: Full list of parameters, bias acceptance rate $q$ and Jensen-Shannon divergence (JSD) for each model presented in the main text Fig. 3, 4 and 6 . We obtain the values for $\bar{H}_{\min }$ and $\bar{H}_{\max }$ from MIN model $\bar{H}$ distribution for each $N$ and $I_{\min }$. The JSD is calculated as the distance between the $C_{\text {TRANS }}, \bar{H}$ or $\bar{F}$ distributions obtained by the MIN model and the TRANS, HAR or FIF model.

\begin{tabular}{|c|c|c|c|c|c|c|c|c|c|}
\hline Model & $N$ & $I_{\min }$ & $n$ & $w$ & $\bar{H}_{m i n}$ & $\bar{H}_{\max }$ & $\beta$ & $q$ & JSD \\
\hline RAN & 4 & 0 & & & & & 0.0 & $1.0 \mathrm{e}+00$ & \\
\hline RAN & 5 & 0 & & & & & 0.0 & $1.0 \mathrm{e}+00$ & \\
\hline RAN & 6 & 0 & & & & & 0.0 & $1.0 \mathrm{e}+00$ & \\
\hline RAN & 7 & 0 & & & & & 0.0 & $1.0 \mathrm{e}+00$ & \\
\hline RAN & 8 & 0 & & & & & 0.0 & $1.0 \mathrm{e}+00$ & \\
\hline RAN & 9 & 0 & & & & & 0.0 & $1.0 \mathrm{e}+00$ & \\
\hline MIN & 4 & 80 & & & & & 0.0 & $7.9 \mathrm{e}-01$ & \\
\hline MIN & 5 & 80 & & & & & 0.0 & $5.9 \mathrm{e}-01$ & \\
\hline MIN & 6 & 80 & & & & & 0.0 & $3.7 \mathrm{e}-01$ & \\
\hline MIN & 7 & 80 & & & & & 0.0 & $2.0 \mathrm{e}-01$ & \\
\hline MIN & 8 & 80 & & & & & 0.0 & $8.4 \mathrm{e}-02$ & \\
\hline MIN & 9 & 80 & & & & & 0.0 & $2.5 \mathrm{e}-02$ & \\
\hline HAR & 4 & 80 & & 20 & 14.0 & 43.98 & 3.0 & $5.6 \mathrm{e}-02$ & 0.12 \\
\hline HAR & 5 & 80 & & 20 & 15.0 & 41.67 & 7.0 & $2.3 \mathrm{e}-03$ & 0.38 \\
\hline HAR & 6 & 80 & & 20 & 16.0 & 39.47 & 13.0 & $2.9 \mathrm{e}-05$ & 0.66 \\
\hline HAR & 7 & 80 & & 20 & 17.0 & 37.57 & 9.5 & $1.7 \mathrm{e}-04$ & 0.51 \\
\hline HAR & 8 & 80 & & 20 & 18.0 & 35.58 & 9.0 & $6.4 \mathrm{e}-05$ & 0.42 \\
\hline HAR & 9 & 80 & & 20 & 18.0 & 31.84 & 14.0 & $2.6 \mathrm{e}-06$ & 0.71 \\
\hline TRANS & 4 & 80 & 2 & & & & 200.0 & $7.8 \mathrm{e}-02$ & 0.48 \\
\hline TRANS & 5 & 80 & 2 & & & & 284.8 & $1.3 \mathrm{e}-02$ & 0.62 \\
\hline TRANS & 6 & 80 & 2 & & & & 1666.7 & $3.9 \mathrm{e}-05$ & 0.82 \\
\hline TRANS & 7 & 80 & 2 & & & & 471.4 & $2.0 \mathrm{e}-04$ & 0.77 \\
\hline TRANS & 8 & 80 & 2 & & & & 412.5 & $6.2 \mathrm{e}-05$ & 0.76 \\
\hline TRANS & 9 & 80 & 2 & & & & 500.0 & $6.0 \mathrm{e}-06$ & 0.79 \\
\hline FIF & 4 & 80 & & 20 & & & 2000.0 & $3.2 \mathrm{e}-03$ & 0.60 \\
\hline FIF & 5 & 80 & & 20 & & & 2000.0 & $1.2 \mathrm{e}-03$ & 0.61 \\
\hline FIF & 6 & 80 & & 20 & & & 4000.0 & $3.9 \mathrm{e}-06$ & 0.71 \\
\hline FIF & 7 & 80 & & 20 & & & 4000.0 & $9.4 \mathrm{e}-07$ & 0.72 \\
\hline FIF & 8 & 80 & & 20 & & & 4000.0 & $9.7 \mathrm{e}-08$ & 0.72 \\
\hline FIF & 9 & 80 & & 20 & & & 4000.0 & $9.1 \mathrm{e}-09$ & 0.73 \\
\hline
\end{tabular}

There remains the possibility that there is an optimal interval size, if one considers the effect of the vocal motor constraint theory, which states that larger intervals are harder to sing [50. By combining these two theories, of vocal mistuning and motor constraint, one may predict an optimal interval size. However we do not currently see a solid foundation upon which to construct such an analysis. One main issue is that singing and listening abilities vary widely across individuals, thus the optimal interval size would likely be best described by a broad distribution. Such a distribution is unlikely to be specific enough to account for the diversity of scales by itself, but it may be useful to consider this in tandem with other theories.

\section{Model parameter sensitivity}

In total, four tunable parameters are used in our generative models of scales: $I_{\min }, n, w$ and $\beta . I_{\min }$ is the minimum interval size allowed in a scale such that generated scales with any interval smaller than $I_{\text {min }}$ are rejected. $n$ is a parameter in the cost function for the TRANS model; higher $n$ corresponds to larger deviations from a compressible interval template being penalized more heavily than smaller deviations. $w$ is the window size for the HAR and FIF model templates; higher $w$ corresponds to a greater tolerance for errors when perceiving consonant intervals. $\beta$ controls the strength of any applied bias; the same value of $\beta$ in different models does not result in the same strength of the bias. We report instead $\log _{10} q$, where $q$ is the fraction of generated scales that are accepted by the model, such that increasing $\beta$ reduces $q$. $q$ is not a perfect indicator of bias strength, but it is easy to measure and scales with model performance better than $\beta$. The $q$ values for the different models (not reported in the main paper) are shown in Table 4 . For each model we generated $S=10^{4}$ scales. Two metrics are used to characterize the performance of the models: $d_{\mathrm{I}}$ and $f_{\mathrm{D}} \cdot d_{\mathrm{I}}$ is the distance between the model and empirical $I_{\mathrm{A}}$ distributions. $f_{\mathrm{D}}$ is the fraction of scales from the database which are predicted by the model.

For the TRANS model, increasing $n$ appears to improve the results, albeit slightly. For both the HAR and FIF models maximum performance is attained when $w=20-30 \sqrt{2} \mathrm{~B}-\mathrm{C})$. For all models shown there is a clear optimal $\log _{10} q$, which corresponds to an optimal $\beta$. For all the models, performance is greatest for $I_{\min }=80-90$ cents $2 \mathrm{D}$ ).

We chose to present results in Fig. 2 for the FIF model using a different cost function than the one presented in the main text:

$$
C_{\mathrm{FIF}}=1-(N \bar{F})^{2},
$$

where $\bar{F}$ is the fraction of intervals that are fifths, and $N$ is the number of notes in a scale. This is purely for purposes of illustration. When we use the cost function shown in the main text, the acceptance rate is too low to clearly show the optimal values of $\log _{10} q$, i.e., the extrema in Fig. 23 would not be clear. In Eq. $2, N$ is factor that normalizes the range of the cost function so that for all $N, 0 \leq C_{\mathrm{FIF}} \leq 1$. A full explanation of how the cost function affects the results is illustrated in Fig. 13,14 and 15

\section{Packing of fifths produces interval mixing rules for scales}

Our generative model arranges intervals randomly into scales. To see if scales from the database are arranged randomly, we calculated the fraction of small $\left(\mathbf{S}: I_{\mathrm{A}}<(1-x) 1200 / N\right)$, medium $\left(\mathbf{M}:(1-x) 1200 / N \leq I_{\mathrm{A}} \leq(1+x) 1200 / N\right)$ and large intervals $\left(\mathbf{L}: I_{\mathrm{A}}>(1+x) 1200 / N\right)$, using $x=0.2$. We use these formulae to define size categories since we are interested in relative sizes rather than absolute sizes. Using this fractions, we calculated the probability that certain intervals are found adjacent to each other by mixing randomly, and compared these results with the DAT and model-generated scales (Fig. 33. We find that the DAT scales are much more likely to have small intervals placed with large intervals and vice versa than random mixing would predict. 
A
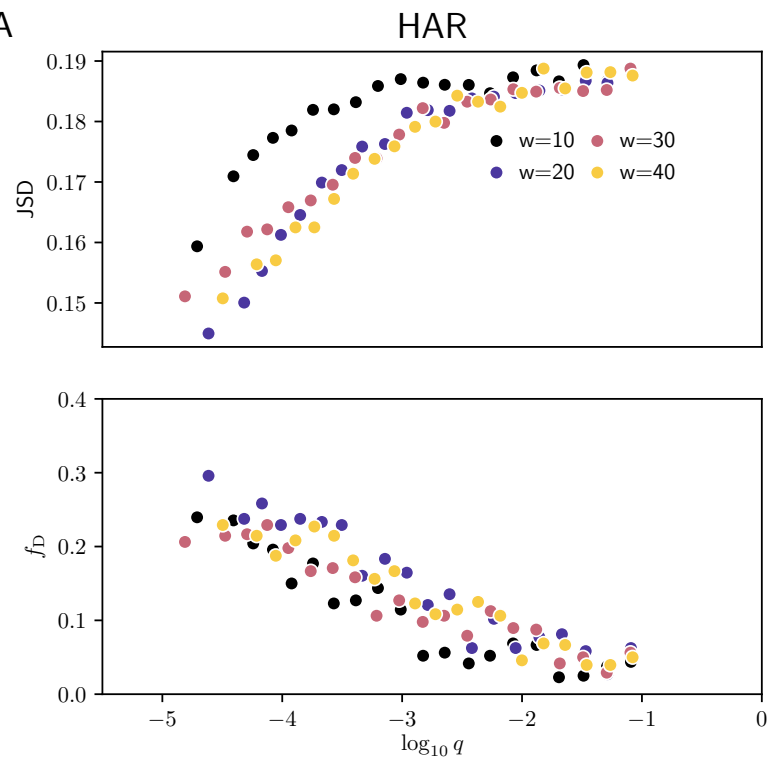

C
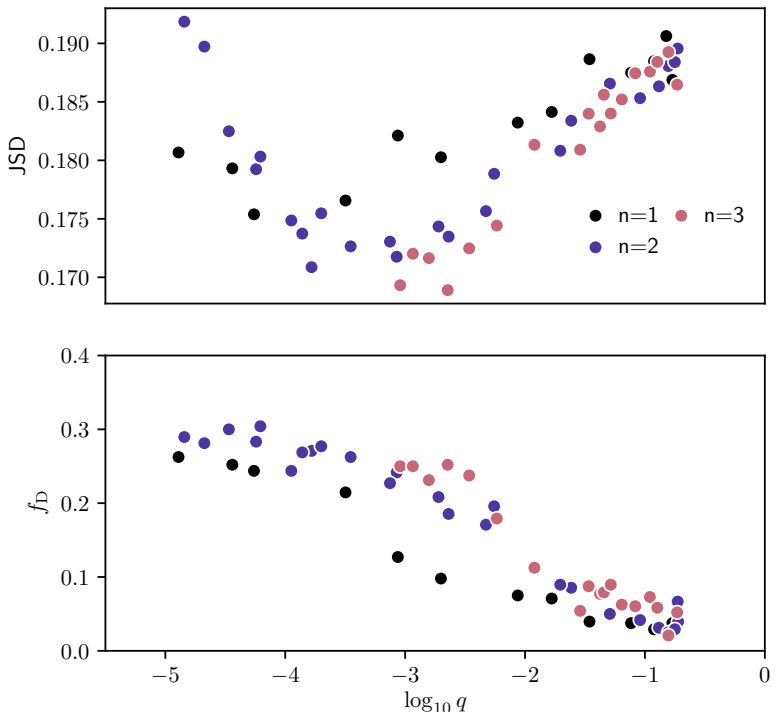

B
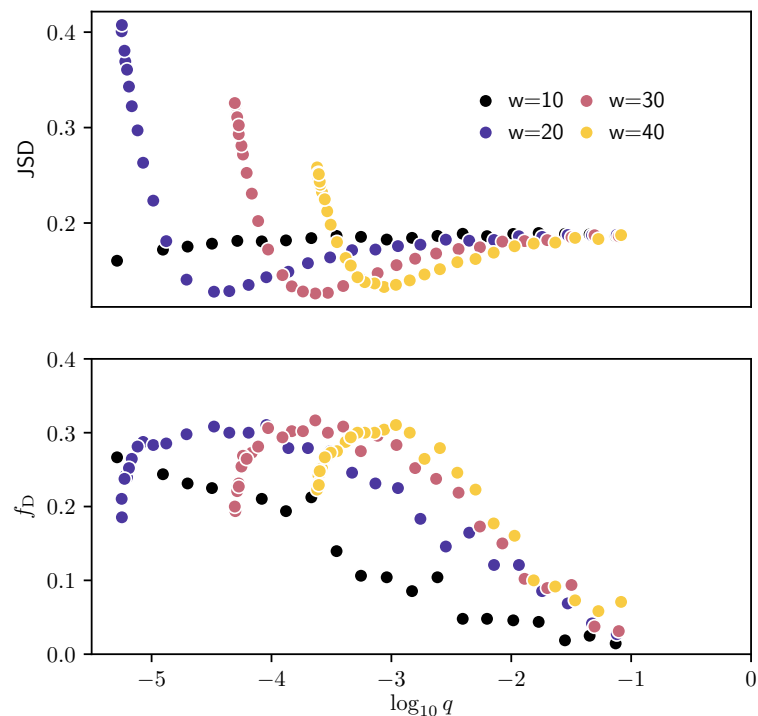

D
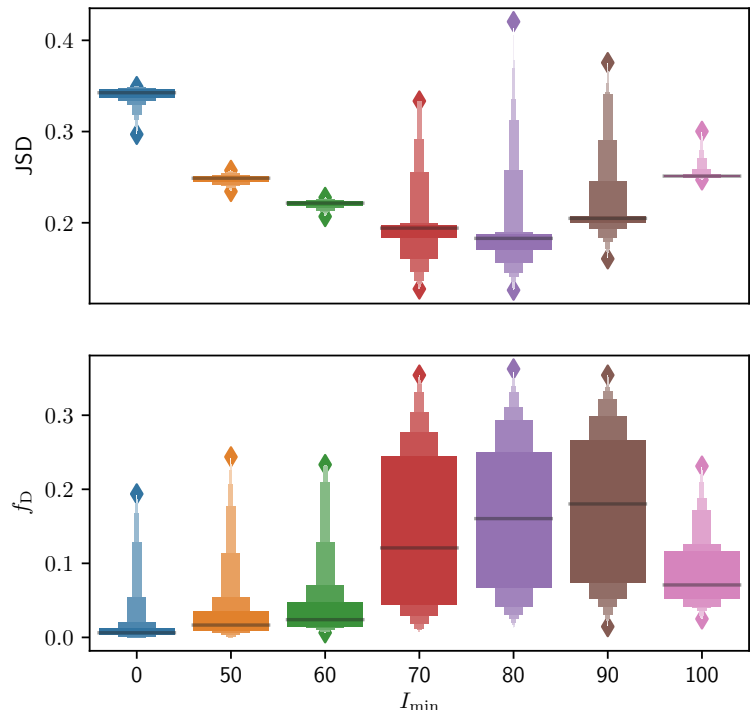

Figure 2: A-C: The Jensen-Shannon divergence between model and empirical distributions, JSD, and the fraction of real scales found by the models, $f_{\mathrm{D}}$, as a function of the fraction of generated scales, $q$, that are accepted by the model. This is reported for the three models (A) HAR, (B) FIF and (C) TRANS, for $I_{\min }=80$. D: The distributions of $d_{\mathrm{I}}$ and $f_{\mathrm{D}}$ for all models as a function of the minimum interval size, $I_{\min }$. This includes results from all models. Note that the results for $I_{\min }=[70,80,90]$ appear qualitatively different to the results for other values of $I_{\min }-$ this is mainly due to differences in sampling. A-D: Results are only shown for $N=7$ for clarity. 
A
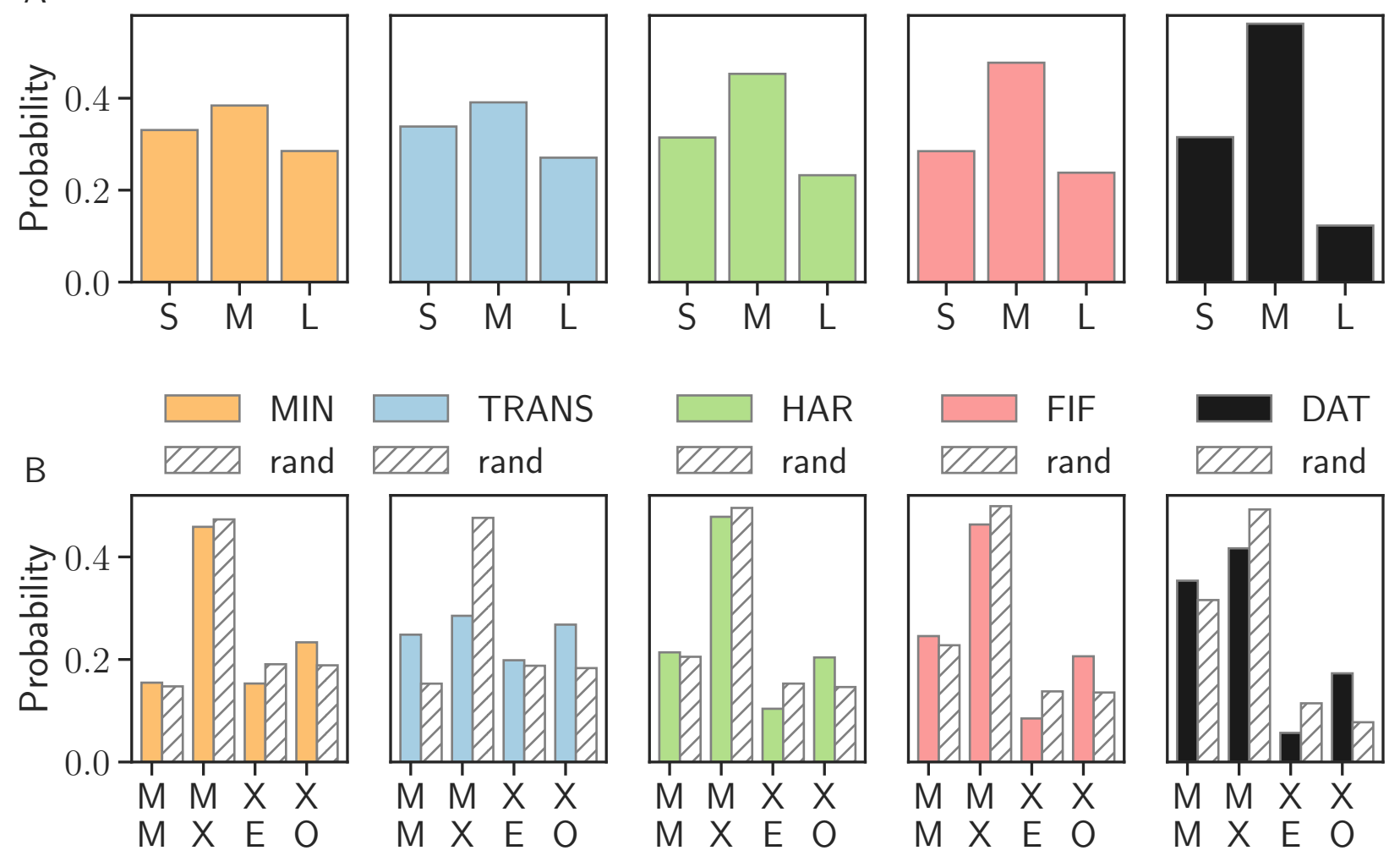

Figure 3: A: The distribution of interval sizes (S: small, M: medium, L: large) in populations for MIN, TRANS, HAR, FIF, and DAT. B: The probability that interval sizes are found adjacent to each other in populations, compared with the probabilities of random pairing; $\mathbf{M}-\mathbf{M}$ (medium interval beside a medium interval), $\mathbf{M}-\mathbf{X}$ (X: extreme, which includes $\mathbf{S}$ and $\mathbf{L}$ ), $\mathbf{X}-\mathbf{E}$ (E: equivalent, which includes S-S or $\mathbf{L}-\mathbf{L}), \mathbf{X}-\mathbf{O}$ (O: opposite, which includes $\mathbf{S}-\mathbf{L}$ and $\mathbf{L}-\mathbf{S})$. We calculate probabilities without considering the order in which the intervals are chosen. A-B: Results are only shown for $N=7$ for clarity.

To investigate the influence of the above effect, we rearranged the intervals in our model-generated scales by biasing them so that they are well-mixed. Scales are considered well-mixed if the sum of two consecutive intervals is close to the average sum of two intervals, $2 \times 1200 / N$. This means that small intervals are placed beside large intervals so that their combined size approximates that of two medium intervals. To arrange scales so that they are mixed, we calculate the cost function $C_{\text {mix }, j}$ for all unique permutations, $M$, of a set of intervals as

$$
C_{\text {mix }, j}=\left[\frac{1}{N} \sum_{i}^{N}\left(I_{i}+I_{i+1}-\frac{2400}{N}\right)^{2}\right]^{\frac{1}{2}},
$$

where $N$ is the number of notes in a scale, $j$ is the scale index, and $I_{i}$ is the $i^{t h}$ pair interval in a scale. When the subscript $i>N$, due to the circular nature of scales, $i \rightarrow i-N$. We normalize $C_{\mathrm{mix}, j}$ to get $\tilde{C}_{\mathrm{mix}, j}$ for each $j$ by dividing by the maximal $C_{\mathrm{mix}, j}$. We then randomly draw a scale, with the probability of a scale $j$ being picked, $P_{\text {mix }, j}$,

$$
P_{\mathrm{mix}, j}=\frac{\exp \tilde{C}_{\mathrm{mix}, j}^{-1}}{\sum_{k=1}^{M} \exp \tilde{C}_{\mathrm{mix}, k}^{-1}} .
$$

Arranging scales in this way results in higher $f_{\mathrm{D}}$ values for the TRANS model, but lower $f_{\mathrm{D}}$ values for the HAR and FIF models (Fig. 4). Thus, mixing scales in this way improves the results for the TRANS model since it does not take interval order into account in its bias. However for the harmonicity models, which do take interval order into account, this method of ordering intervals results in an inferior fit. This indicates that scales are in general well-mixed rather than random, but it is more important that they are ordered such that they maximise the number of fifths, and perhaps to an extent other harmonic intervals.

\section{Correlations between harmonicity models}

There are multiple models of harmonicity, which primarily differ in how they deal with deviations from exact integer ratios and how they weight different harmonics. Using the incon package [51 in $\mathrm{R}$ we tested five models [52 56] for correlations with the model of Gill and Purves (2009) (HAR model) by comparing their predictions for the twelve diatonic intervals. Pearson's $r$ values of $r>=0.75$ indicate that the models are significantly correlated (Fig. 5 A). We compared the HAR and FIF models in the same way and found that they are less correlated (Fig. 5A). For the purposes of this study, however, it is more appropriate to check correlations between the average scores of scales rather than intervals. For a set of scales (MIN model, $N=7, S=10^{4}$ ) we calculate for each scale the average harmonicity score as

$$
\bar{H}=\left[\sum_{i=0}^{N-1} \sum_{j=i+1}^{i+N-1} H\left(I_{i j}, w\right)^{m} / 100^{m-1}\right][N(N-1)]^{-1},
$$




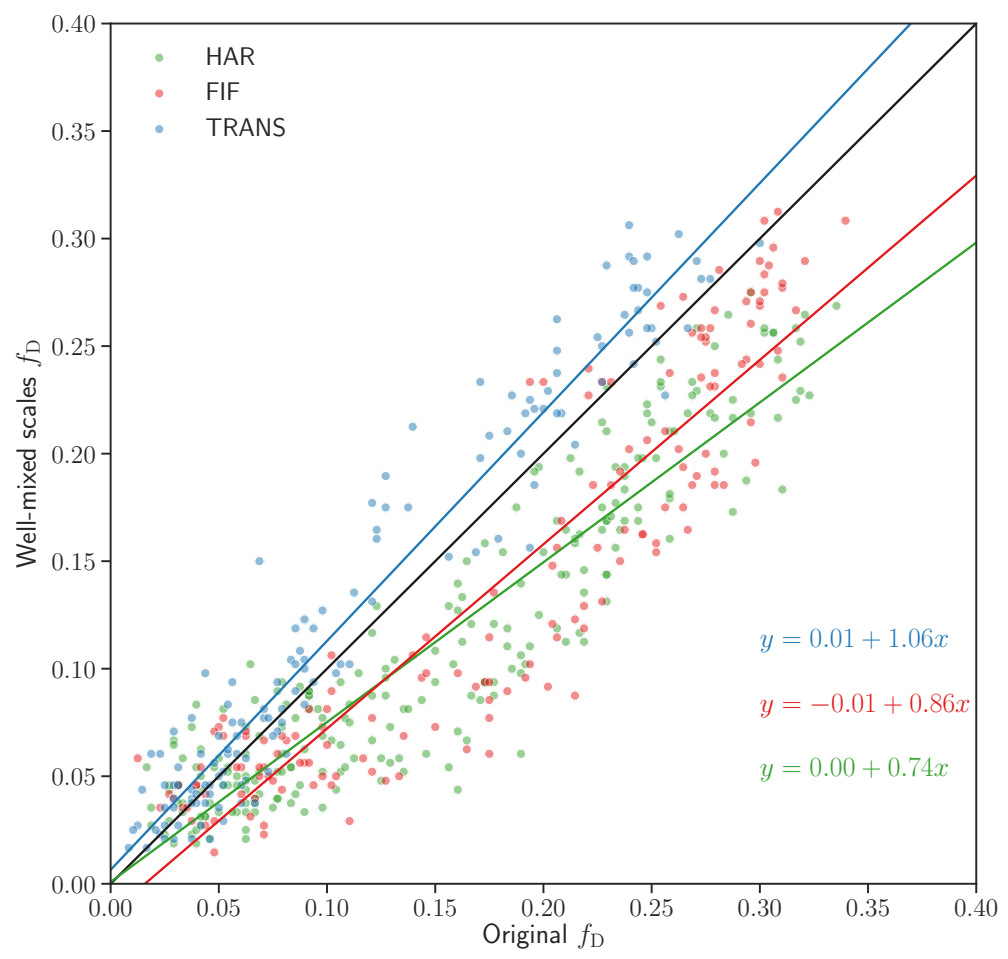

Figure 4: The fraction of real scales $f_{\mathrm{D}}$ originally found by the models plotted against $f_{\mathrm{D}}$ calculated after scales are rearranged with a bias towards being well-mixed; results are shown for the HAR, FIF and TRANS models.

where $H(I)$ is the harmonic similarity score of an interval size $I, w$ is the size of the window in which intervals are considered equivalent, and $I_{i j}$ is the interval between note $i$ and note $j$. The index $i=0$ refers to the starting note of the scale and $j$ takes into account the circular nature of scales (if $j>N$ then note $j$ is an octave higher than note $j-N$ ). The $100^{m-1}$ term is a normalization factor to ensure that $S_{\mathrm{HAR}} \leq 100$. Note that in the HAR model presented in the main text we use $m=1$, while $m=2$ and $m=3$ correspond to $\mathrm{HAR}^{2}$ and $\mathrm{HAR}^{3}$ in the main text Fig. 5. The harmonic similarity score of an interval is calculated from its frequency ratio expressed as a fraction.

$$
H(I)=\frac{x+y-1}{x y} \times 100
$$

where $x$ is the numerator and $y$ is the denominator of the fraction. The harmonic similarity template is produced by creating a grid of windows of maximum size $w$ centred about the intervals that have the largest $H$ values. An interval expressed in cents is allocated to the window with the highest $H$ value that is within $w / 2$ cents. For the same set of scales we calculate the fraction of fifths as

$$
\left.\bar{F}=\left[\sum_{i=0}^{N-1} \sum_{j=1}^{i+N-1} \operatorname{FIF}\left(I_{i j}, w\right)\right][N(N-1)]\right]^{-1},
$$

where $\operatorname{FIF}(I)=1$ if $|I-702| \leq w / 2$. We show that for if $w>=10$ there is a strong correlation between average HAR and FIF scores for scales (Fig. 5B). This correlation increases further as $m$ increases. As $m \rightarrow \infty$ the HAR model becomes a linear function of the FIF model.

\section{Scales that are found, and not found by the models}

We show additional counts of scales that are found or not found by the three models across three categories (Fig. 6): 16 clusters that are based on the scales' adjacent interval sets; scale type ('theory' or 'measured'); geographical region (continent). The fact that the models do not particularly depend on scale type or geographical region are good indicators that the models capture properties of scales that are general. In particular we note that only scales from the 'theory' type are known to have been at one point explicitly based on fifths.

\section{Correlations between transmittability and harmonicity models}

We tested for correlations between the transmittability and harmonicity models by calculating the correlations between cost functions in two populations of scales: DAT scales $(S=742)$ and MIN scales $\left(S=10^{4}\right)$. The results for the MIN scales indicate that in general the models are not correlated (Fig. 7). However, after scales have been selected by humans (DAT scales) there is a significant $\left(p<10^{-30}\right)$ correlation between the TRANS and FIF models, while there is a weak but significant $\left(p<10^{-3}\right)$ correlation between the TRANS and HAR models.

\section{Tritone intervals are scarce due to packing of fifths}

The frequency of tritone intervals in our database, $f_{t}$, is calculated as a function of $N$ such that

$$
f_{t}=\frac{1}{N(N-1) S_{N}} \sum_{i}^{S_{N}} t_{i}
$$


A

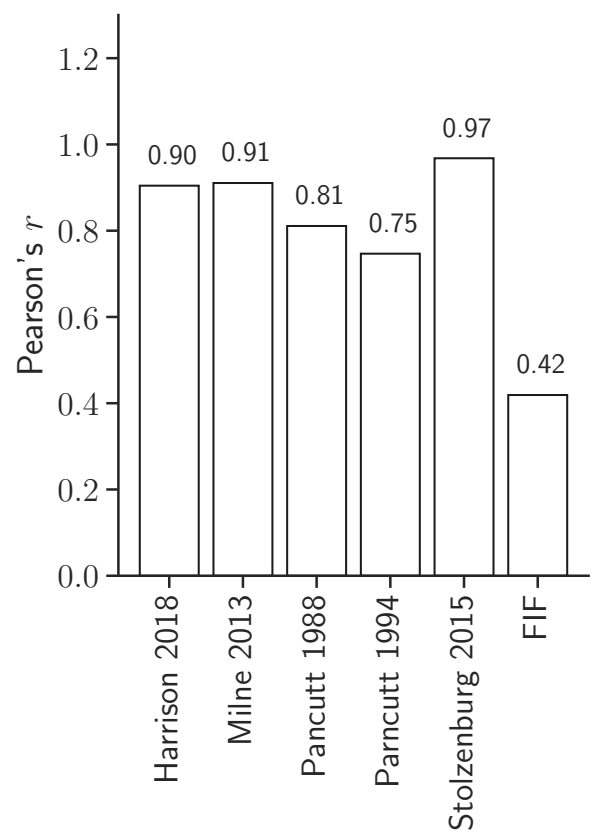

B

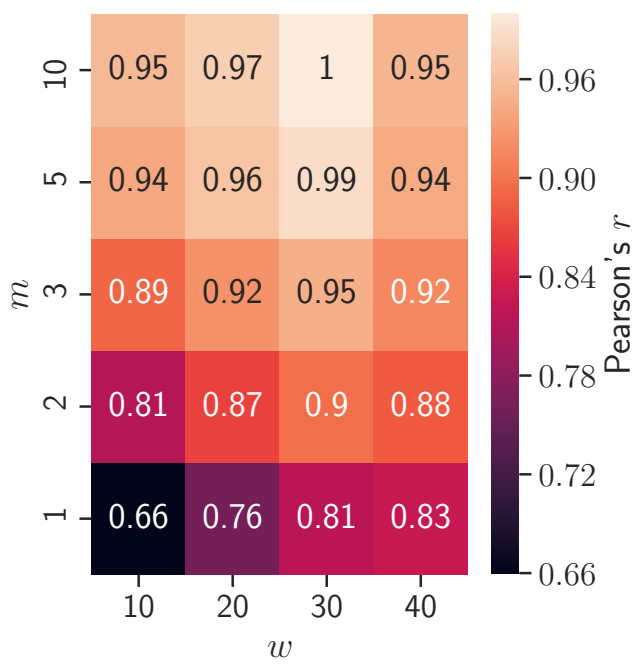

Figure 5: A: Correlations between the model of Gill and Purves (2009) and six other harmonicity models. B: Correlations between the average harmonicity score, $\bar{H}$, and the fraction of fifths, $\bar{F}$, for a population of scales $\left(\mathrm{MIN}\right.$ model, $\left.N=7, S=10^{4}\right)$. For each comparison, both models use the same value of $w$.

where $N$ is the number of notes in a scale, $t_{i}$ is the total number of tritone intervals in scale $i$, and $S_{N}$ is the sample size of $N$ note scales. We consider any interval which is $600 \pm 20$ cents to be a tritone, given that accepted tritone frequency ratios $10 / 7$ and $7 / 5$ correspond to 618 and 583 cents respectively. The database (DAT) scales shows that the fraction of tritone intervals increases linearly with $N$ (Fig. 8). The model that best reproduces this trend is the FIF model.

\section{Thai and Gamelan scales are variable individually, but coherent as an ensemble}

Pelog scales are unlikely to be predicted by any model since they have high costs compared to other scales (Fig. 10A). The TRANS model fits pelog scales better than the other models.

The Thai tuning is considered to be equidistant, and intervals from an ensemble of Thai tunings can indeed be approximated by a Gaussian distribution with a mean $\mu=1200 / 7$ (Fig. 10B). However, intervals within individual scales can deviate wildly from this theoretical ideal, with ranges of up to 96 cents observed in our database. The tunings of Gamelan slendro and pelog scales exhibit similar behaviour (Fig. [10 C).

\section{Results are robust to sub-sampling of the database}

We repeat our analysis for the best performing models for each model shown in the main text Fig. 5 on sub-samples of the database. We use three types of sub-samples: all of the 'theory' scales; all of the 'measured' scales; bootstrapped resamples. We created 10 bootstrapped resamples for each size: $n=0.4 S, n=0.6 S$ and $n=0.8 S$; where $S$ is the size of the sample from which the resamples are drawn. We only used data for $N=5$ and $N=7$ due to the small sample sizes of other $N$. Comparing the results from main text Fig. 5 to the results of the sub-samples we found that under most cases the results do not qualitatively differ (Fig. 11). The main exception is that for the sub-sample which only includes 'measured' scales, the harmonicity models have relatively low values of $d_{\mathrm{I}}$. This indicates that when only considering 'measured' scales, the harmonicity models do not reproduce the adjacent interval distributions more accurately than chance (MIN model).

\section{Hexatonic scales are not so rare as the database suggests}

Using several databases, we studied the distribution of $N$ unique notes used in folk melodies across cultures. The databases include: Essen folk song collection (Chinese and European) [57]; KernScores humdrum database (Native American, Polish, European) 58; the Meertens tune collection (Dutch) [59]; Uzan Hava humdrum database (Turkish) 60. While all cultures shown here have a preference for either 5 or 7 notes in their songs, 6 note songs are consistently the second most frequent (Fig. 12). This effectively means that six note scales are actually quite prevalent, despite the fact that they are rarely counted as scales.

\section{Effect of the functional form of the cost function}

Since we are using a Boltzmann distribution

$$
P=\min \{1, \exp (-\beta C)\},
$$

for each of the three models we need a cost function which approaches zero for the type of scales that the model promotes. In the case of the TRANS model the cost function already satisfies this condition $-C_{\text {TRANS }}$ is a measure of the deviations from a compressible template, such that a scale with $C_{\text {TR ANS }}=0$ maximises lossless compression. For the HAR and FIF models, however, we want to maximise respectively the average harmonic score $\bar{H}$ and the fraction of fifths $\bar{F}$. For purposes of illustration, in the following we consider how to choose a cost function for the HAR model. 
A
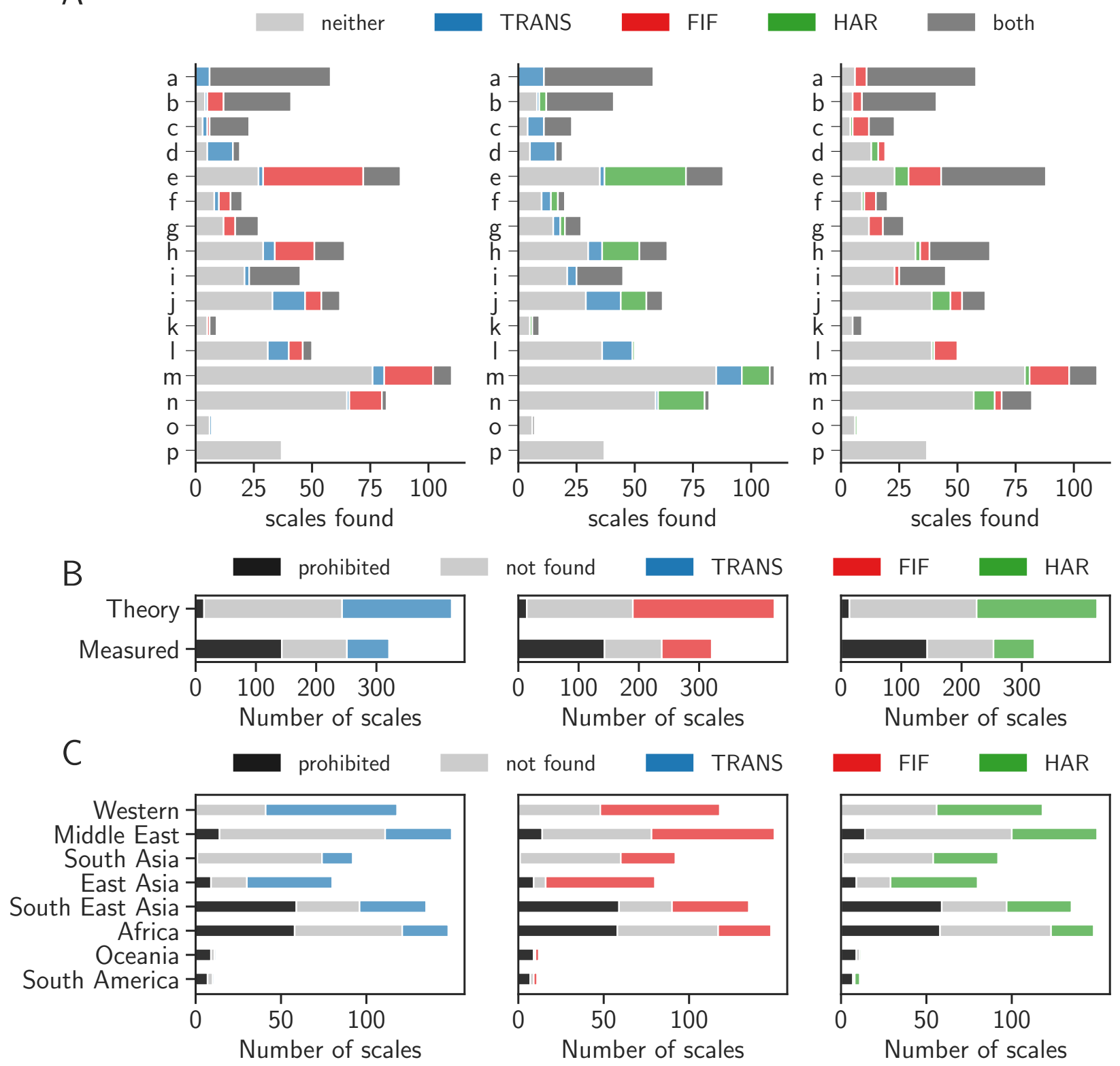

Figure 6: A: Comparison of the scales found by two models for three sets: TRANS and FIF; TRANS and HAR; HAR and FIF. Stacked bars indicate what scales are found by: neither model; only one of either model; both models. B: Number of 'theory'/'measured' scales that are not found, 'prohibited' (it is not possible to find them due to the hard constraints of the model), and found by each model. C: Number of scales from each continent that are not found, 'prohibited' and found by each model. 

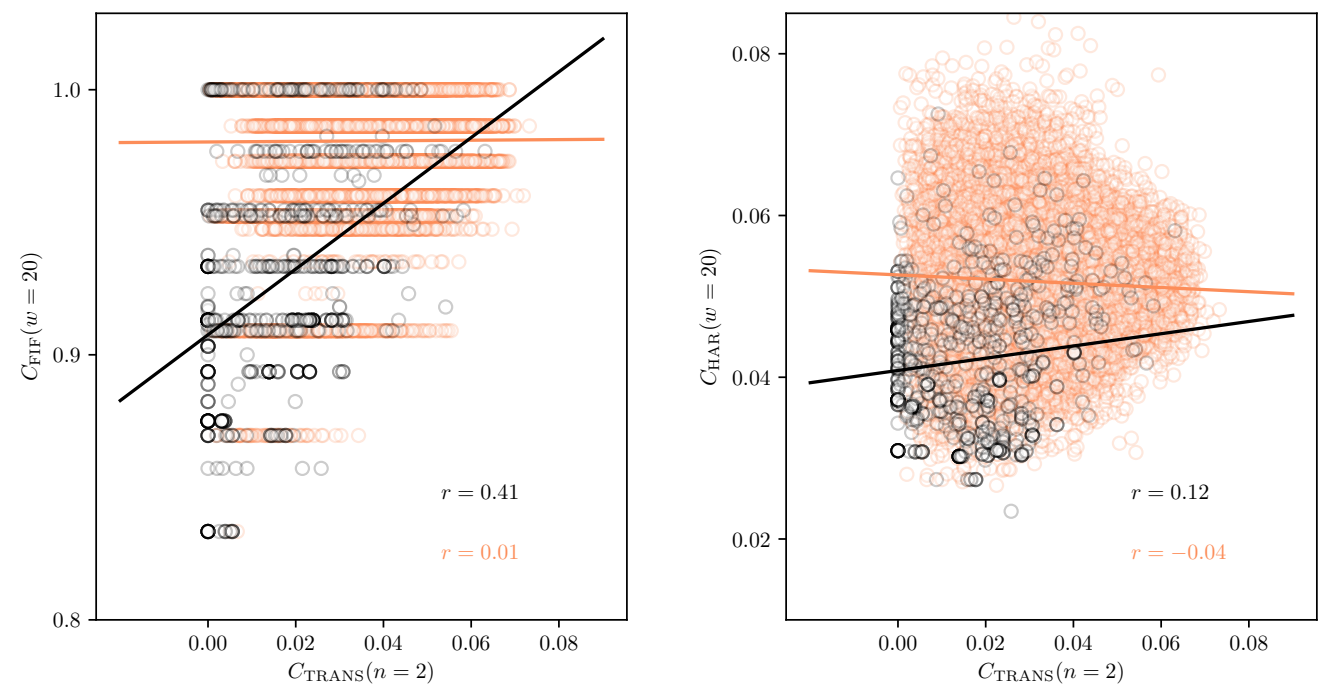

Figure 7: Correlations between transmittability and harmonicity cost functions for two sets of data: DAT scales $(S=742$, black) and MIN scales $\left(S=10^{4}\right.$, orange). Pearson's $r$ is indicated in the plots for each correlation.

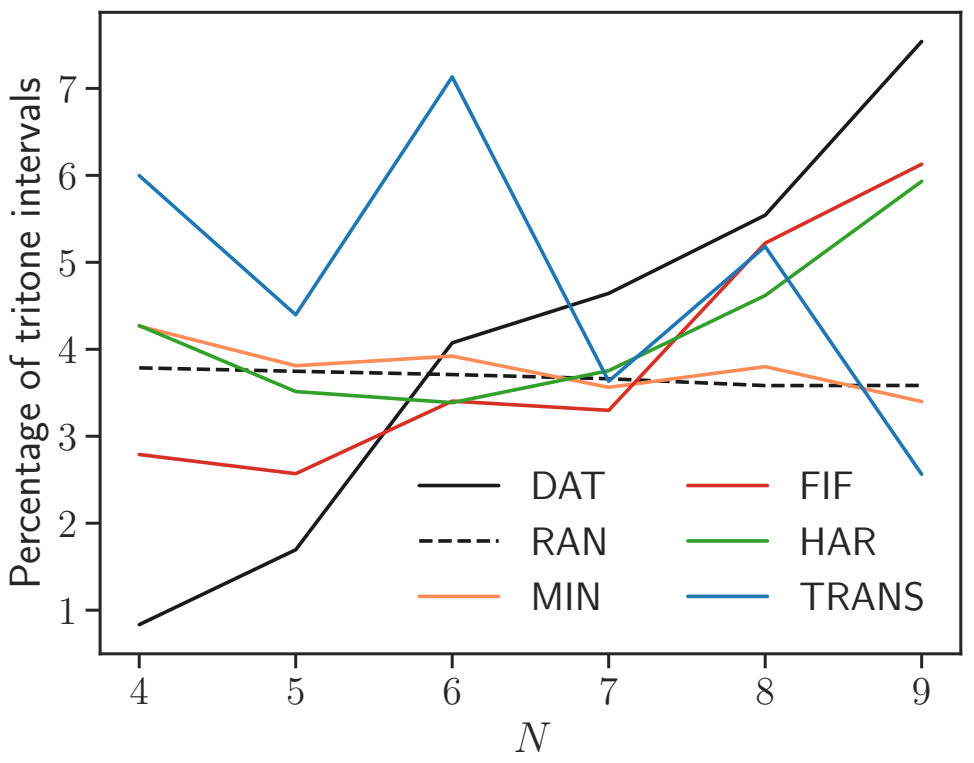

Figure 8: The percentage of tritone intervals found in $N$ note scales in our database and in scales generated by the models reported in Fig. 3. 
A
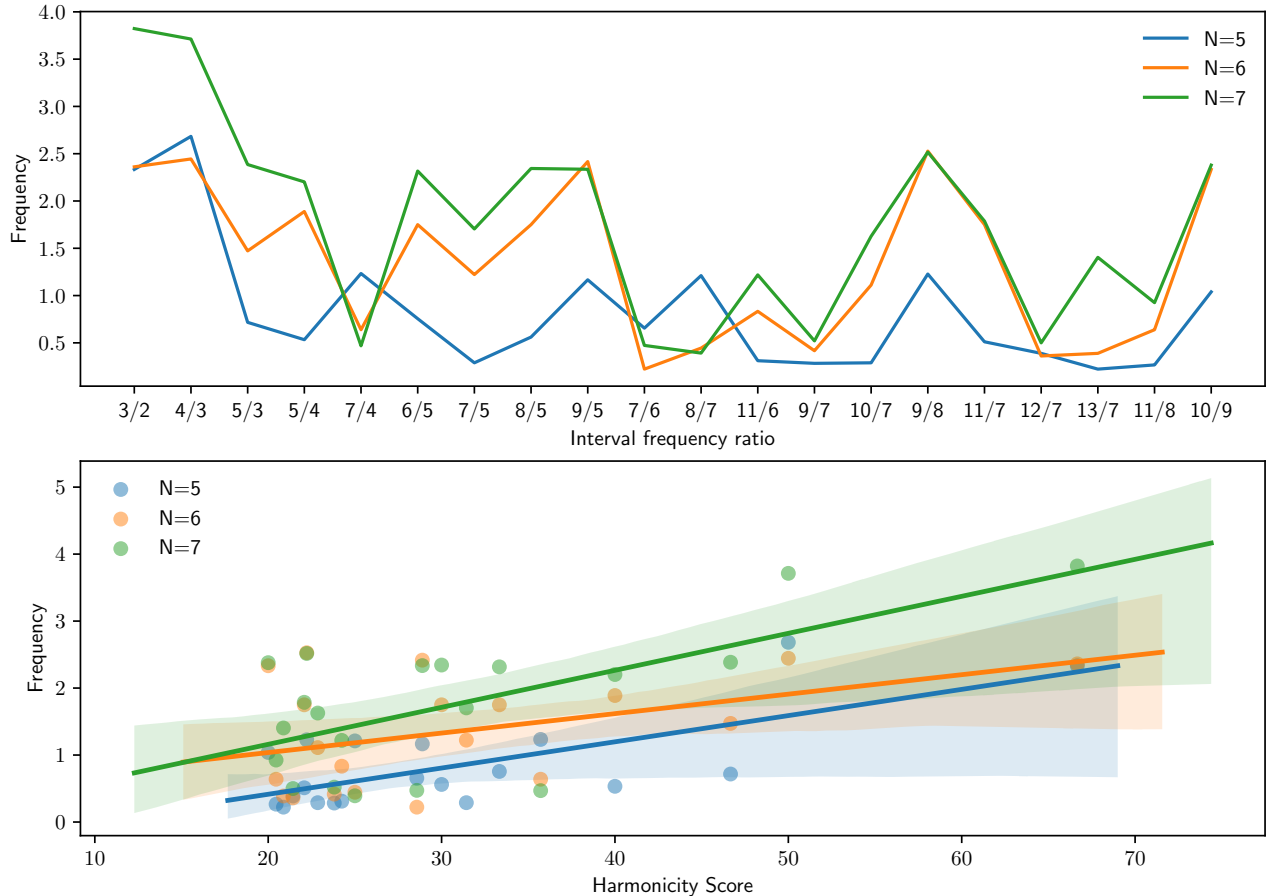

Figure 9: A: The frequency of intervals found in DAT scales, where the intervals are ordered according to their harmonicity score. B: Frequency of intervals found in DAT scales, as a function of harmonicity. Regression lines are shown with shaded regions indicating $95 \%$ confidence intervals $(N=5, r=0.70, p<0.005 ; N=6, r=0.43, p>0.05 ; N=7, r=0.65, p<0.005$; SI Fig. 9).

The exact functional form of the cost function is not necessarily important. We are interested instead in how it affects the results of the model. There are two main effects of the cost function: the acceptance rate and the selectivity. The acceptance rate is simple to measure,

$$
\text { acc }=\sum P_{\text {acc }}(\bar{H})
$$

where $P_{\text {acc }}(\bar{H})$ is the probability that a scale with $\bar{H}$ is accepted. One measure for selectivity is the relative entropy between generated and accepted $\bar{H}$ distributions. However two different sets of distributions can give the same relative entropy. In particular, non-linear changes to the cost function will result in a different $\bar{H}$ distribution for the same relative entropy (linear changes are cancelled out by $\beta$ which is varied independently). Thus there is no obvious optimal way of constructing a cost function.

Our approach is to design a cost function which allows us to optimize the bias strength via $\beta$ for the best model performance. We want to maximize the acceptance rate, while maximizing the selectivity, so that the model can reach the optimum selectivity in reasonable time. We study the effect of the functional form analytically, given a probability distribution of $\bar{H}$. In the following we use the $\bar{H}$ distribution resulting from the MIN model with $N=7$ and $S=10^{4}$ (Fig. $13 \mathrm{~A}$ ). As a result we have

$$
P_{\mathrm{acc}}=\sum P_{\mathrm{MIN}}(\bar{H}) P(\beta, C(\bar{H}))
$$

where $P_{\text {MIN }}(\bar{H})$ is the probability of generating a scale with the average harmonicity score $\bar{H}$. By specifying a cost function $C(\bar{H})$ and $\beta$ we can then get the analytical probability distribution of $\bar{H}$ for the accepted scales. We use the Jensen-Shannon divergence, JSD, as a measure of selectivity. Using this method we study how the selectivity and acceptance rate change depending on the functional form of the bias.

There are two basic ways of defining such a cost function for the HAR model such that as $\bar{H}$ increases the cost decreases:

$$
C_{1}=1-\bar{H} / A
$$

and

$$
C_{2}=1 /(A+\bar{H})
$$

where $A$ is a constant. Due to the form of the Boltzmann distribution if $C<0$ then $P=1$ regardless of $\beta$. This means that in general one should choose $A$ such that $C>=0$ for all $\bar{H}$ : for $C_{1}$ this is when $A \geq \bar{H}_{\max }$; for $C_{2}$ this is when $A \geq-\bar{H}_{\text {min }}$. We can choose to violate this principle, with variable consequences. For $C_{1}$, the consequences are not so severe as the $\bar{H}$ distribution is positively skewed (Fig. $13 \mathrm{~A} \mathrm{left)}$ - i.e., if $A$ is slightly lower than $\bar{H}_{\max }$ little will change because the probability of generating scales with $\bar{H}$ close to $\bar{H}_{\max }$ is small, and the scales which end up with a negative cost are those with high $\bar{H}$ (the scales we wish to prioritize). This is because the form of $C_{1}$ acts to make scales with high $\bar{H}$ have low cost. However for $C_{2}$, the consequences are severe (Fig. $13 \mathrm{~A}$ right). In this case, the form of $C_{2}$ acts to penalize scales with low $\bar{H}$, i.e., $C_{2} \rightarrow 0$ only as $\bar{H} \rightarrow \infty$, which is impossible as $\bar{H}$ is bounded by $\bar{H}_{\max }$. If $A \leq-\bar{H}_{\min }$ then scales with low $\bar{H}$ have $P=1$ due to the change of sign in $C_{2}$.

If we vary $A$ in the other direction $\left(A>\overline{\bar{H}}_{\max }\right.$ for $C_{1}, A>-\bar{H}_{\min }$ for $\left.C_{2}\right)$, we get a lower acceptance rate for a given selectivity (Fig. $13 \mathrm{~B}-\mathrm{C}$ ). For $C_{1}$ changing $A$ in this way does not qualitatively alter the selectivity because the change is linear (Fig. $13 \mathrm{~A}$ left - the orange and purple lines are the same). However for $C_{2}$ changing $A$ in this way does qualitatively alter the selectivity (the orange and purple lines are not the same in Fig. 13A right). Thus, each cost function has a clear optimum value of $A$ : at $A=\bar{H}_{\max }$ for $C_{1}$, and at $A=-\bar{H}_{\min }$ for $C_{2}$.

We can further optimize the cost function with an additional parameter $m$ :

$$
C_{3}=1-(\bar{H} / A)^{m},
$$




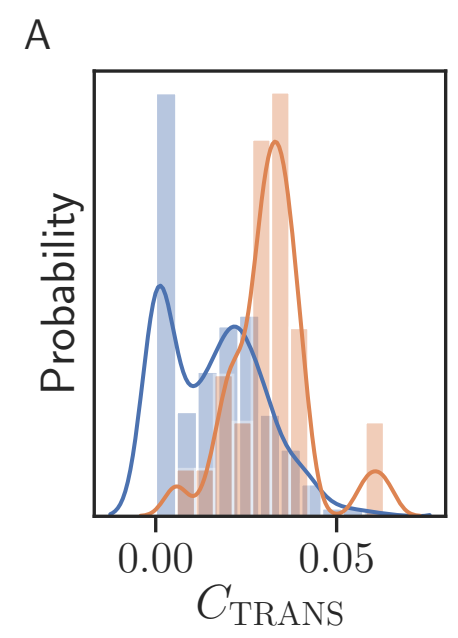

Pelog
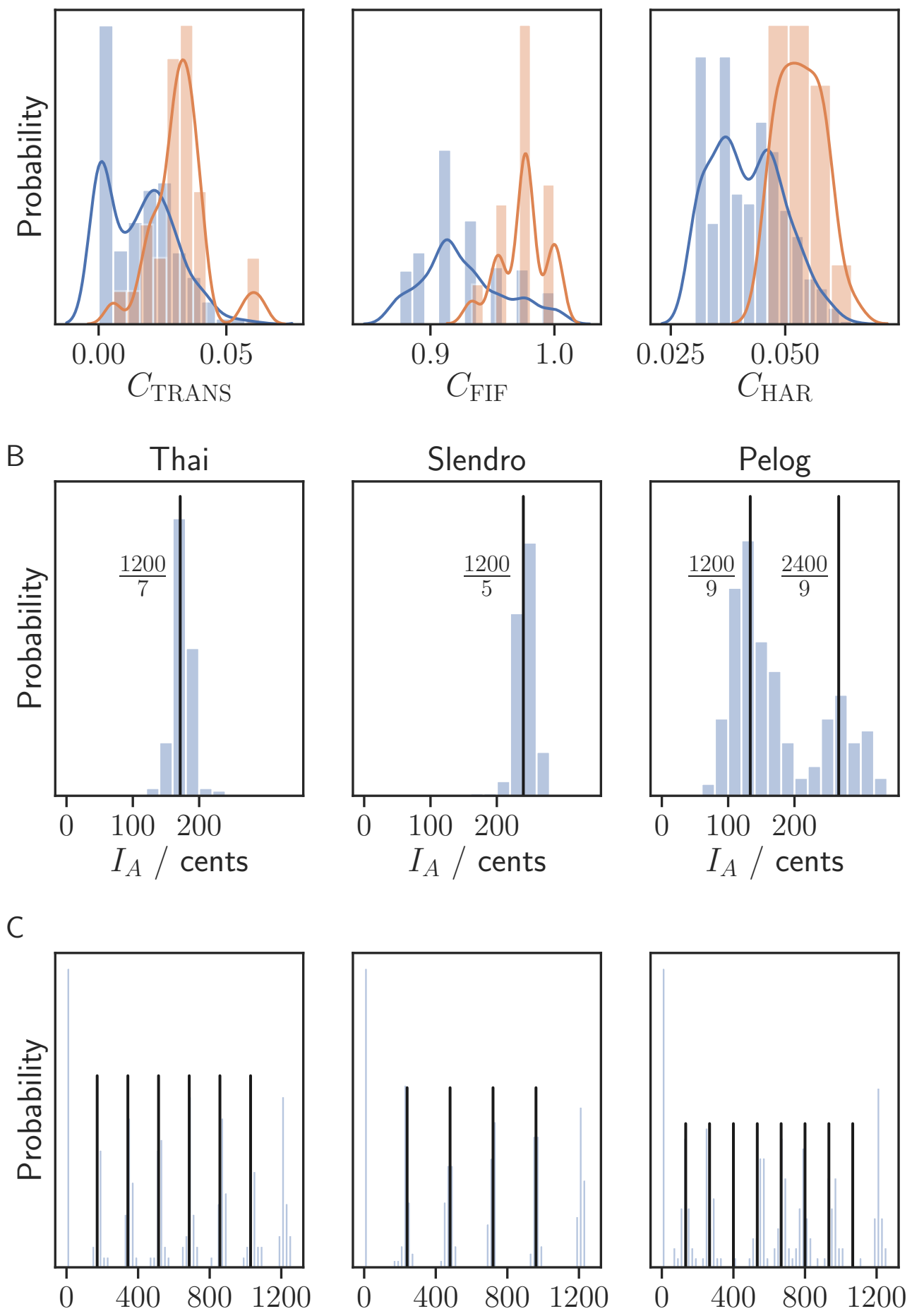

Notes in scale / cents Notes in scale / cents Notes in scale / cents

Figure 10: A: The distributions of the cost functions for pelog scales (orange) and all DATscales (blue: including the pelog scales). B-C: The probability distributions of pair intervals, $I_{\mathrm{A}},(\mathrm{B})$ and scale notes (C) for three types of scales: the 7 note Thai tuning, the 5 note Gamelan slendro scale, and the 7 note Gamelan pelog scale. Lines correspond to exactly equidistant scales for 7 -tet, 5-tet and 9-tet tunings. 

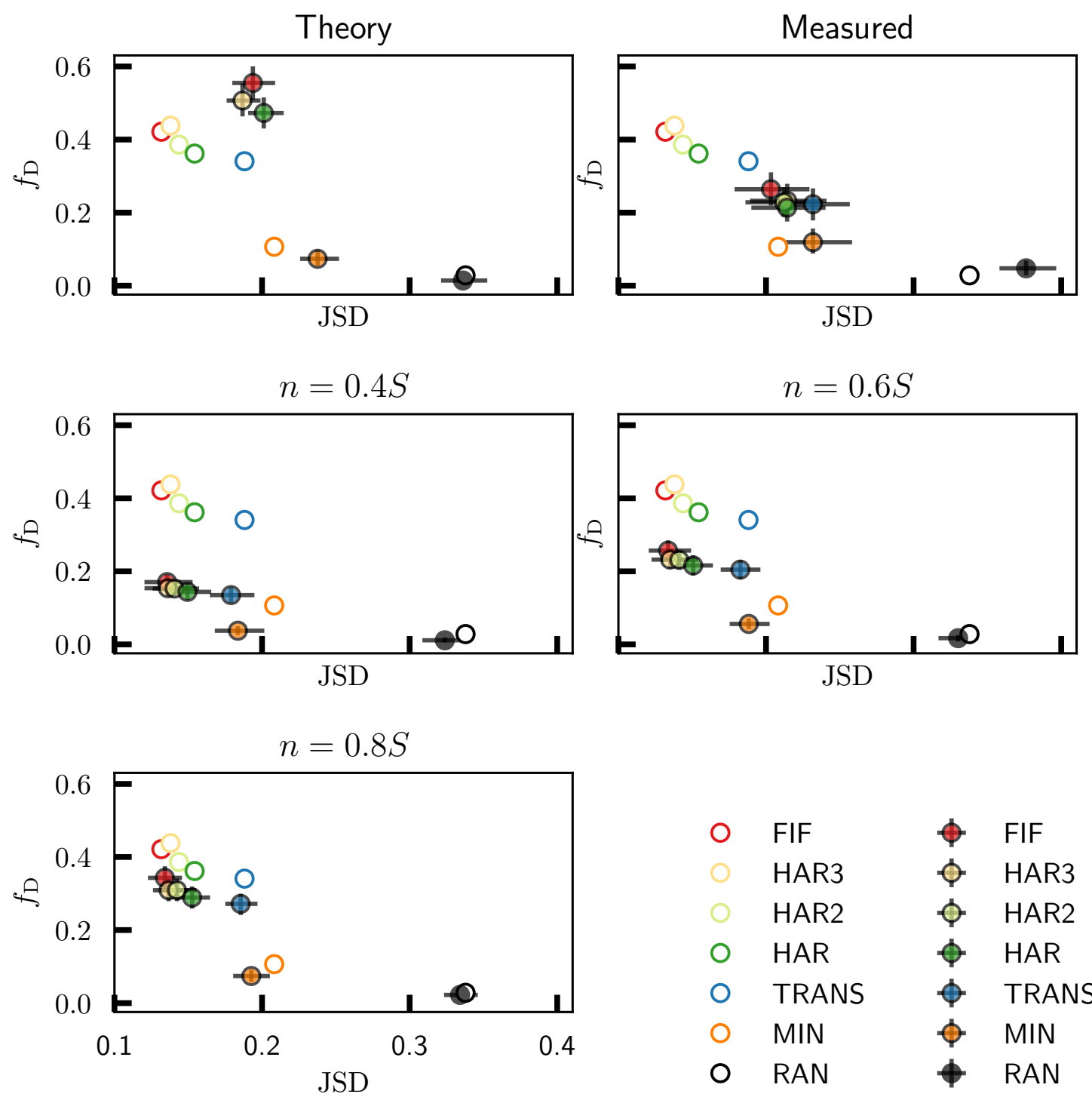

$\begin{array}{ll}0 & \text { FIF } \\ 0 & \text { HAR3 } \\ 0 & \text { HAR2 } \\ 0 & \text { HAR } \\ 0 & \text { TRANS } \\ 0 & \text { MIN } \\ 0 & \text { RAN }\end{array}$

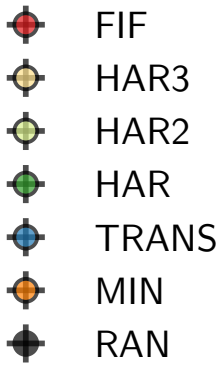

Figure 11: Results for models tested against resampled data: results are shown for Theory (only the 'theory' scales), Measured (only the 'measured' scales) and three sizes of bootstrapped samples. Whiskers indicate 95\% confidence intervals. Results for the best performing models using the full data set, DAT, are shown in each plot as empty circles. 


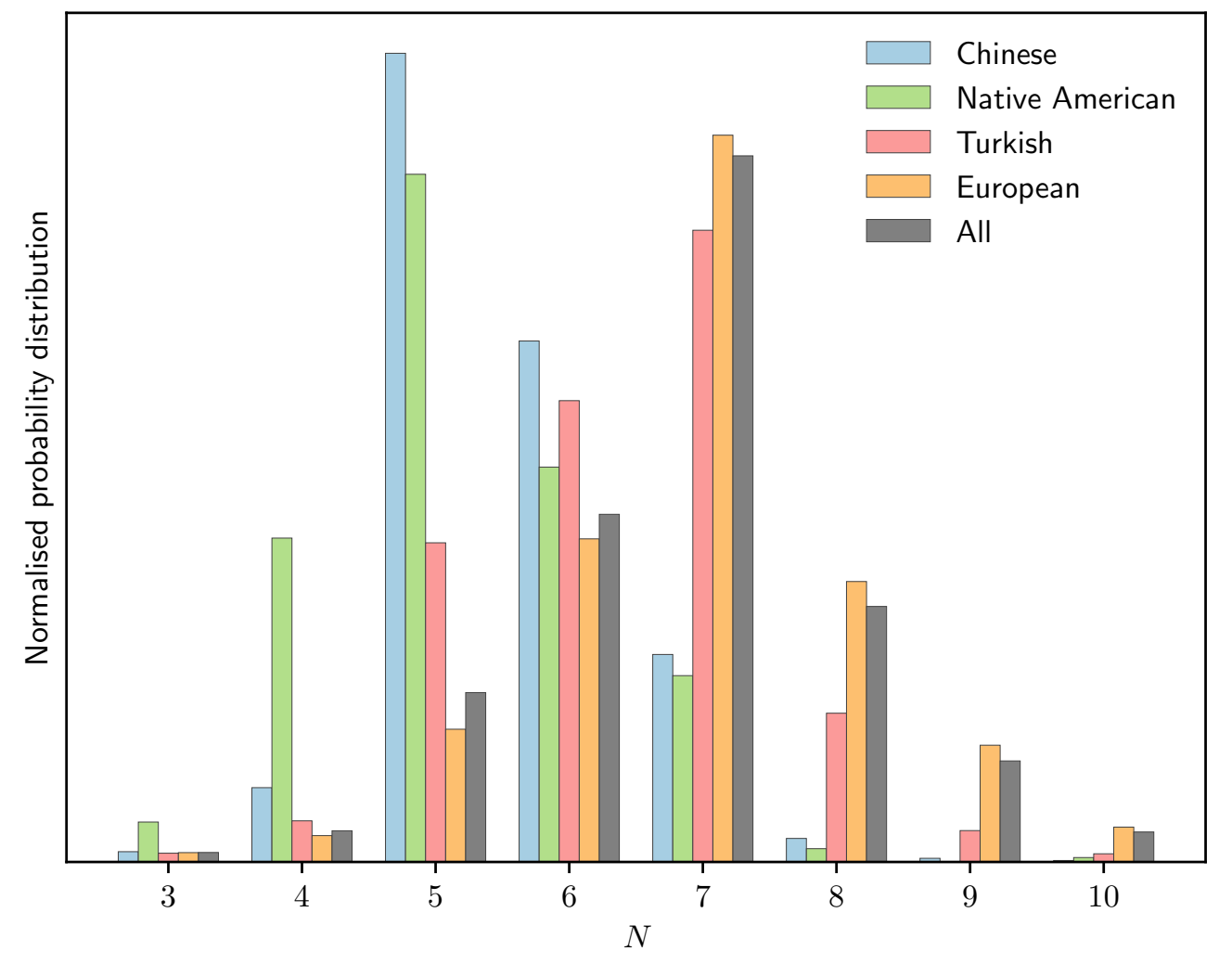

Figure 12: The probability distributions of number of notes $N$ in folk songs from different cultures (Chinese, Native American, Turkish, European).

$$
C_{4}=1 /(A+\bar{H})^{m} .
$$

Changing $m$ has opposing effects for $C_{3}$ and $C_{4}$ (Fig. 14B-C). For $C_{3}$, decreasing $m$ results in a converging, increasing acceptance rate, while increasing $m$ results in a diverging, decreasing acceptance rate. For $C_{4}$, increasing $m$ results in a converging, increasing acceptance rate, while decreasing $m$ results in a diverging, decreasing acceptance rate. Crucially, any changes to $m$ will result in different $\bar{H}$ distributions for a fixed selectivity. For all the theoretical $\bar{H}$ distributions in Fig. $14 \mathrm{~A}$ the a selectivity is set at 0.5 , but they differ considerably. This should result in different results in our simulations. We investigated the effect of $m$ for $C_{3}$ and $C_{4}$ on the performance of the corresponding models (Fig. 15). We find that changing $m$ does change the performance of the models under some conditions. For $C_{3}\left(C_{4}\right)$ the results improve with decreasing (increasing) $m$, however this effect appears to converge at the level of performance of the HAR model presented in the main text. 

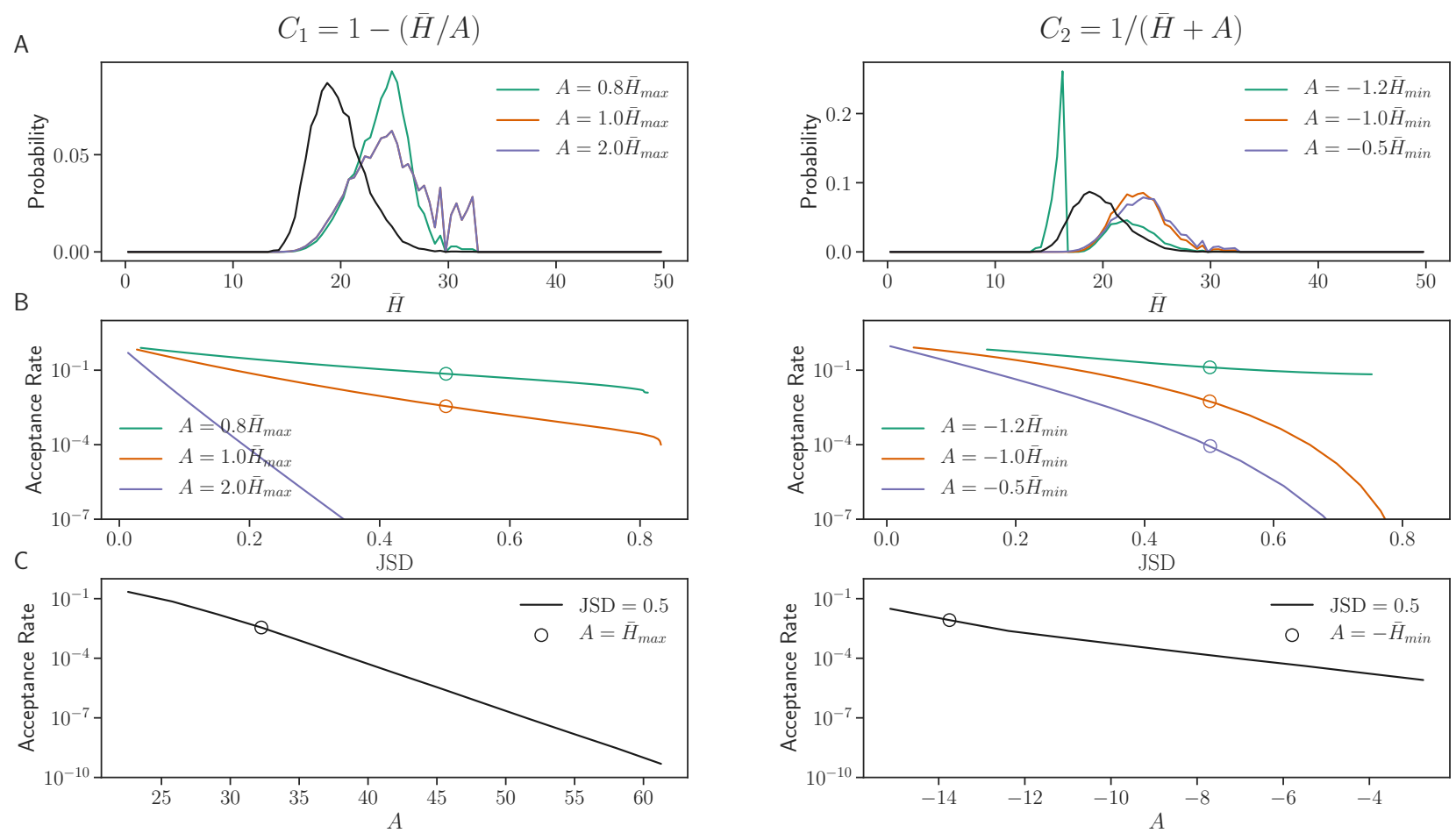

Figure 13: Effect of changing $A$ in the cost functions $C_{1}$ (left) and $C_{2}$ (right). A: Probability distribution of $\bar{H}$ for a population of $N=7$ scales accepted by the MIN model (black line). Theoretical probability distributions of $\bar{H}$ accepted by models with the cost functions $C_{1}$ (left) and $C_{2}$ (right). Values of $A$ correspond to: $A$ chosen so that some scales are always accepted (green line), $A$ chosen so that the acceptance is maximized, and $C>=0$ (orange line), $A$ chosen so that the acceptance is sub-optimal and $C>=0$ (purple line). $\beta$ is chosen so that the selectivity (JSD) is 0.5 . B: Acceptance rate vs. selectivity (JSD) for the same values of $A$ as in A. Increasing $\beta$ decreases acceptance rate, and increases selectivity (JSD). Circles correspond to a selectivity (JSD) of 0.5. C: Dependence of the acceptance rate on $A$. Selectivity is constant at 0.5. Circles correspond to the points where $A$ maximizes selectivity (JSD) while maintaining $C>=0$.

A
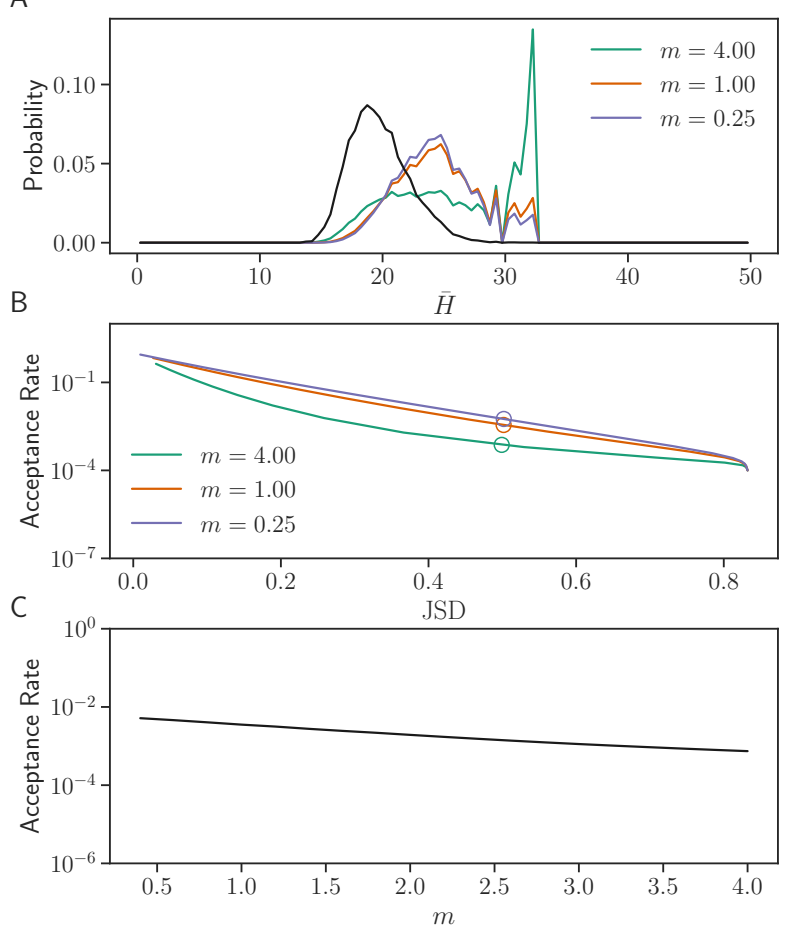

$$
C_{4}=1 /(\bar{H}+A)^{m}
$$
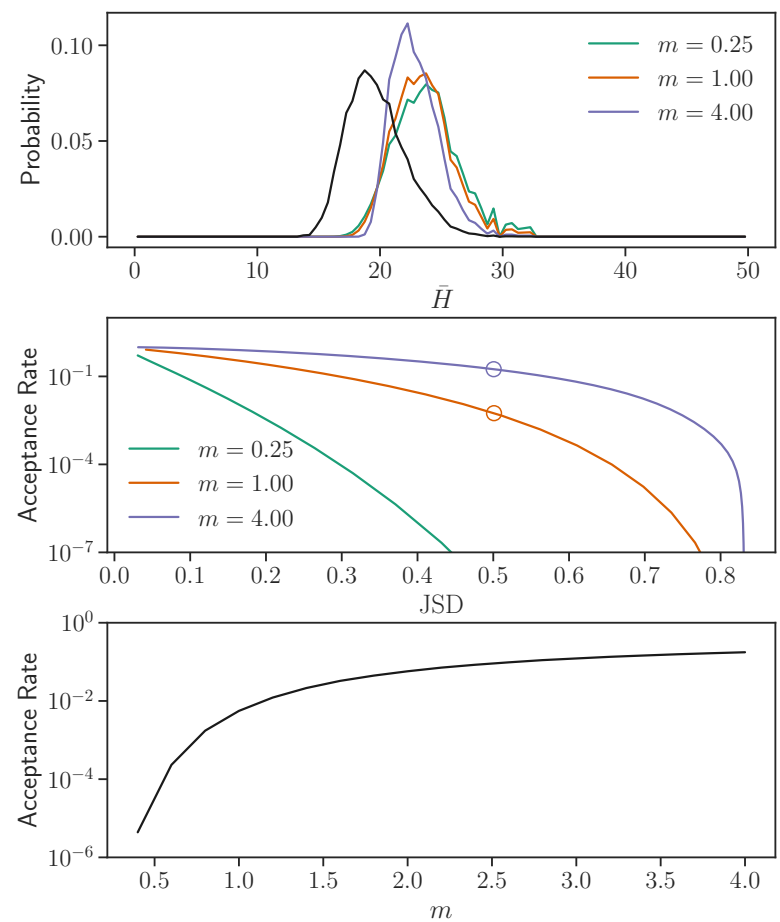

Figure 14: Effect of changing $m$ in the cost functions $C_{3}$ (left) and $C_{4}$ (right). A: Probability distribution of $\bar{H}$ for a population of $N=7$ scales accepted by the MIN model (black line). Theoretical probability distributions of $\bar{H}$ accepted by models with the cost functions $C_{3}$ (left) and $C_{4}$ (right). Values of $m$ correspond to: $m$ chosen so that the acceptance rate increases (green line), $m=1$ (orange line), $m$ chosen so that the acceptance rate decreases (purple line). $\beta$ is chosen so that the selectivity (JSD) is 0.5 . B: Acceptance rate vs. selectivity (JSD) for the same values of $m$ as in A. Circles correspond to a selectivity (JSD) of 0.5 . C: Dependence of the acceptance rate on $m$. Selectivity is constant at 0.5 . 

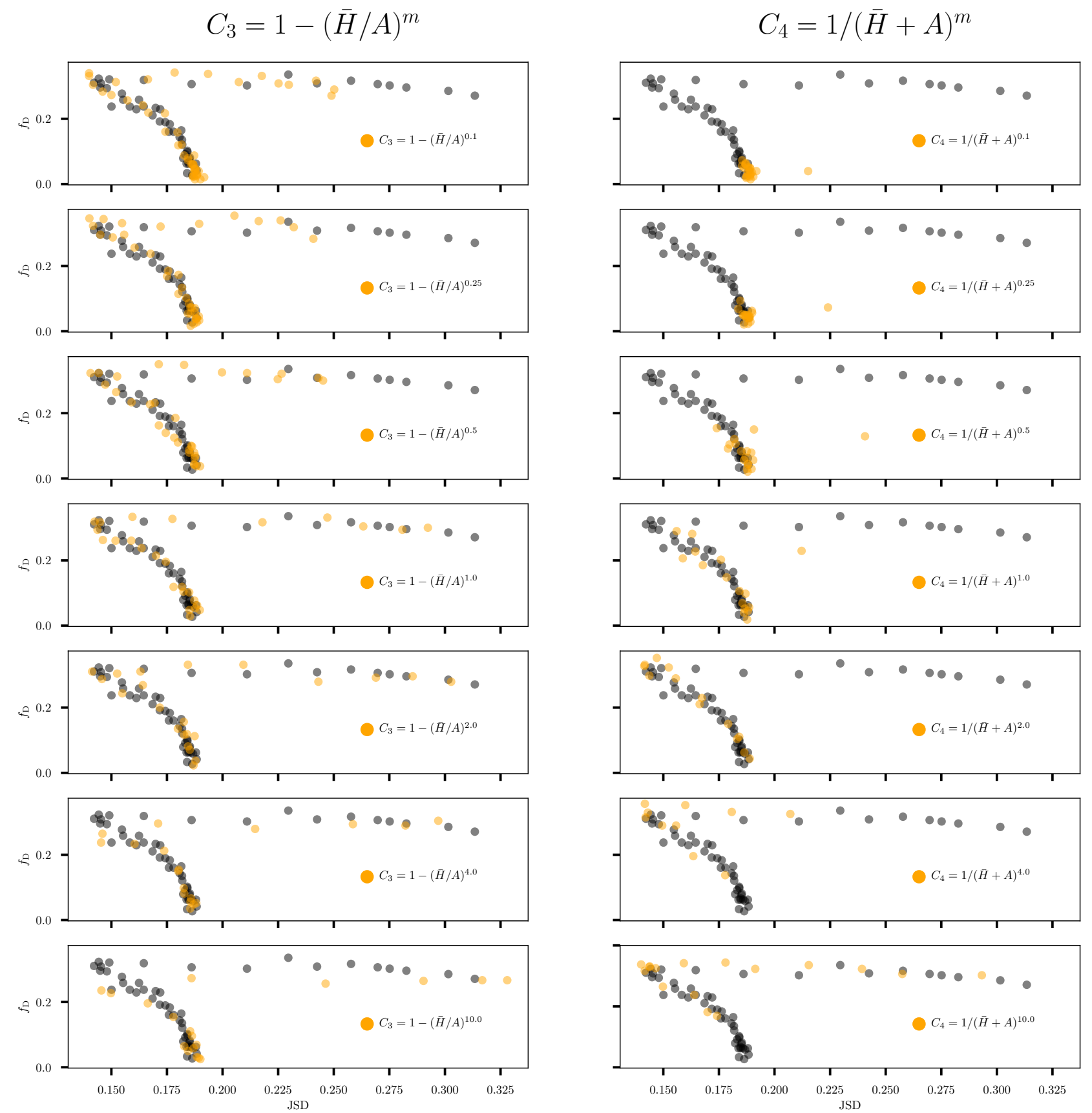

Figure 15: Effect of changing $m$ on model performance for the cost functions $C_{3}$ (left) and $C_{4}$ (right), for $m=$ $0.1,0.25,0.5,1,2,4,10$. For each subplot we report the model performance as the Jensen-Shannon divergence between adjacent interval distributions, JSD, and the fraction of scales found by the model $f_{\mathrm{D}}$. The black circles mark the results of the main HAR model. Increasing $\beta$ results in $f_{\mathrm{D}}$ increasing while $d_{\mathrm{I}}$ decreases until the point of optimal results $($ top-left corner of each plot), after which $d_{\mathrm{I}}$ sharply increases. After the optimal point, the fact that $d_{\mathrm{I}}$ decreases while $f_{\mathrm{D}}$ is relatively constant indicates that when $\beta$ is higher than the optimum, the cost function is specialized for a subset of scales. 
Table 5: Goodness-of-fit statistics for how well the model distributions fit the DAT distributions in the main text Fig. 3. For each model and each $N$ we show: sample size $S$ for DAT scales of size $N$; Jensen-Shannon divergence, JSD, between DAT and model $I_{\mathrm{A}}$ distributions (mean and $95 \%$ confidence intervals); two sample Cramér-von Mises criterion (CvM) for each set of $I_{\mathrm{A}}$ distributions (mean and 95\% confidence intervals); fraction of real scales found, $f_{\mathrm{D}}$, (mean and 95\% confidence intervals).

\begin{tabular}{|c|c|c|c|c|c|c|c|c|}
\hline Model & $N$ & $S$ & JSD & $\mathrm{CI}$ & $\mathrm{CvM}$ & CI & $f_{D}$ & $\mathrm{CI}$ \\
\hline \multirow[t]{7}{*}{ RAN } & 4 & 10 & 0.33 & $(0.26,0.40)$ & 0.74 & $(0.47,1.03)$ & 0.49 & $(0.20,0.80)$ \\
\hline & 5 & 180 & 0.32 & $(0.30,0.34)$ & 14.63 & $(12.21,17.42)$ & 0.07 & $(0.03,0.11)$ \\
\hline & 6 & 36 & 0.34 & $(0.28,0.41)$ & 3.60 & $(2.56,4.84)$ & 0.00 & $(0.00,0.00)$ \\
\hline & 7 & 480 & 0.35 & $(0.34,0.36)$ & 48.65 & $(44.60,53.07)$ & 0.01 & $(0.00,0.02)$ \\
\hline & 8 & 29 & 0.30 & $(0.26,0.34)$ & 3.24 & $(2.75,3.78)$ & 0.00 & $(0.00,0.00)$ \\
\hline & 9 & 7 & 0.30 & $(0.27,0.34)$ & 1.23 & $(0.80,1.79)$ & 0.00 & $(0.00,0.00)$ \\
\hline & arithmatic mean & & 0.34 & $(0.33,0.35)$ & 35.34 & $(32.63,38.36)$ & 0.03 & $(0.02,0.04)$ \\
\hline \multirow[t]{7}{*}{ MIN } & 4 & 10 & 0.23 & $(0.15,0.31)$ & 0.44 & $(0.22,0.69)$ & 0.49 & $(0.20,0.80)$ \\
\hline & 5 & 180 & 0.19 & $(0.17,0.23)$ & 6.30 & $(4.37,8.69)$ & 0.27 & $(0.21,0.34)$ \\
\hline & 6 & 36 & 0.25 & $(0.17,0.32)$ & 1.68 & $(0.95,2.53)$ & 0.08 & $(0.00,0.17)$ \\
\hline & 7 & 480 & 0.19 & $(0.18,0.20)$ & 10.93 & $(9.26,12.99)$ & 0.02 & $(0.01,0.04)$ \\
\hline & 8 & 29 & 0.21 & $(0.18,0.25)$ & 2.55 & $(1.46,3.84)$ & 0.00 & $(0.00,0.00)$ \\
\hline & 9 & 7 & 0.31 & $(0.28,0.33)$ & 1.66 & $(0.87,2.30)$ & 0.00 & $(0.00,0.00)$ \\
\hline & arithmatic mean & & 0.20 & $(0.19,0.21)$ & 8.81 & $(7.53,10.24)$ & 0.09 & $(0.07,0.11)$ \\
\hline \multirow[t]{7}{*}{ HAR } & 4 & 10 & 0.22 & $(0.15,0.31)$ & 0.40 & $(0.20,0.68)$ & 0.51 & $(0.20,0.80)$ \\
\hline & 5 & 180 & 0.16 & $(0.13,0.19)$ & 4.69 & $(2.87,7.04)$ & 0.51 & $(0.44,0.58)$ \\
\hline & 6 & 36 & 0.19 & $(0.16,0.25)$ & 0.69 & $(0.36,1.27)$ & 0.25 & $(0.11,0.42)$ \\
\hline & 7 & 480 & 0.14 & $(0.13,0.15)$ & 6.27 & $(4.94,7.90)$ & 0.31 & $(0.27,0.35)$ \\
\hline & 8 & 29 & 0.20 & $(0.17,0.22)$ & 2.60 & $(1.44,4.01)$ & 0.31 & $(0.17,0.48)$ \\
\hline & 9 & 7 & 0.26 & $(0.25,0.28)$ & 1.44 & $(0.59,2.21)$ & 0.57 & $(0.14,0.86)$ \\
\hline & arithmatic mean & & 0.15 & $(0.14,0.17)$ & 5.34 & $(4.34,6.60)$ & 0.36 & $(0.33,0.39)$ \\
\hline \multirow[t]{7}{*}{ TRANS } & 4 & 10 & 0.25 & $(0.18,0.34)$ & 0.49 & $(0.27,0.76)$ & 0.40 & $(0.10,0.70)$ \\
\hline & 5 & 180 & 0.20 & $(0.17,0.23)$ & 6.43 & $(4.42,8.67)$ & 0.47 & $(0.40,0.55)$ \\
\hline & 6 & 36 & 0.20 & $(0.15,0.27)$ & 1.07 & $(0.57,1.75)$ & 0.33 & $(0.19,0.50)$ \\
\hline & 7 & 480 & 0.18 & $(0.17,0.19)$ & 9.04 & $(7.19,11.07)$ & 0.28 & $(0.24,0.32)$ \\
\hline & 8 & 29 & 0.22 & $(0.18,0.24)$ & 2.41 & $(1.38,3.61)$ & 0.48 & $(0.31,0.66)$ \\
\hline & 9 & 7 & 0.29 & $(0.27,0.30)$ & 1.52 & $(0.81,2.14)$ & 0.72 & $(0.43,1.00)$ \\
\hline & arithmatic mean & & 0.19 & $(0.18,0.20)$ & 7.57 & $(6.28,8.95)$ & 0.34 & $(0.31,0.37)$ \\
\hline \multirow[t]{7}{*}{ FIF } & 4 & 10 & 0.25 & $(0.17,0.33)$ & 0.51 & $(0.28,0.77)$ & 0.49 & $(0.20,0.80)$ \\
\hline & 5 & 180 & 0.13 & $(0.10,0.16)$ & 2.41 & $(1.26,4.01)$ & 0.60 & $(0.53,0.67)$ \\
\hline & 6 & 36 & 0.20 & $(0.14,0.26)$ & 1.40 & $(0.78,2.16)$ & 0.30 & $(0.17,0.47)$ \\
\hline & 7 & 480 & 0.13 & $(0.11,0.14)$ & 5.82 & $(4.78,7.14)$ & 0.37 & $(0.33,0.41)$ \\
\hline & 8 & 29 & 0.17 & $(0.14,0.20)$ & 1.57 & $(0.70,2.69)$ & 0.45 & $(0.28,0.62)$ \\
\hline & 9 & 7 & 0.23 & $(0.22,0.25)$ & 0.98 & $(0.40,1.62)$ & 0.72 & $(0.29,1.00)$ \\
\hline & arithmatic mean & & 0.13 & $(0.13,0.15)$ & 4.49 & $(3.72,5.41)$ & 0.43 & $(0.40,0.46)$ \\
\hline
\end{tabular}




\section{References}

[1] M. J. Hewitt. Musical Scales of the World. Note Tree, 2013.

[2] H. Rechberger. Scales and Modes Around the World: The Complete Guide to the Scales and Modes of the World. Fennica Gehrman Ltd., 2018.

[3] K. P. Wachsmann. An equal-stepped tuning in a ganda harp. Nature, 165(4184):40-41, 1950. doi: 10.1038/165040a0.

[4] Gerhard Kubik. Harp music of the azande and related peoples in the central african republic: (part i horizontal harp playing). Afr. Music, 3(3):37-76, 1964.

[5] Edward C. Carterette, Roger A. Kendall, and Sue Carole De Vale. The matepe mbira music of rhodesia. Afr. Music, 4(4): 37-61, 1970. doi: 10.21504/amj.v4i4.1681.

[6] Edward C. Carterette, Roger A. Kendall, and Sue Carole De Vale. The nyanga panpipe dance. Afr. Music, 5(1):73-89, 1971. doi: $10.21504 /$ amj.v5i1.1152.

[7] W. Surjodiningrat, A. Susanto, and P. J. Sudarjana. Tone Measurements of Outstanding Javanese Gamelans in Jogjakarta and Surakarta. Gadjah Mada University Press, 1972.

[8] D. Morton and C. Duriyanga. The Traditional Music of Thailand, volume 8. Univ of California Press, 1976.

[9] Joerg Haeberli. Twelve nasca panpipes: A study. Ethnomusicology, 23(1):57-74, 1979. doi: 10.2307/851338.

[10] Gerhard Kubik. Likembe tunings of kufuna kandonga (angola). Afr. Music, 6(1):70-88, 1980.

[11] W. Van Zanten. The equidistant heptatonic scale of the asena in malawi. Afr. Music, 6(1):107-125, 1980. doi: 10.21504/amj. v6i1.1099.

[12] Hugo Zemp. Melanesian solo polyphonic panpipe music. Ethnomusicology, 25(3):383-418, 1981. doi: 10.2307/851551.

[13] B. A. Aning. Tuning the kora: A case study of the norms of a gambian musician. J. Afr. Stud., 9(3):164, 1982.

[14] Ho Lu-Ting and Han Kuo-huang. On chinese scales and national modes. Asian Music, 14(1):132-154, 1982. doi: 10.2307/ 834047.

[15] G. Kubik. A structural examination of homophonic multi-part singing in east and central africa. Anuario Musical, 39:27, 1984.

[16] Gerhard Kubik. African tone-systems: A reassessment. Yearb. Tradit. Music, 17:31-63, 1985. doi: 10.2307/768436.

[17] Robert Gottlieb. Sudan ii: Music of the blue nile province; the ingessana and berta tribes, 1986.

[18] R. Yu-An, E. C. Carterette, and W. Yu-Kui. A comparison of the musical scales of the ancient chinese bronze bell ensemble and the modern bamboo flute. Percept. Psychophys., 41(6):547-562, 1987. doi: 10.3758/BF03210489.

[19] D. H. Keefe, E. M. Burns, and P. Nguyen. Vietnamese modal scales of the dan tranh. Music Percept., 8(4):449-468, 1991. doi: $10.2307 / 40285522$.

[20] Edward C. Carterette, Roger A. Kendall, and Sue Carole De Vale. Kambazithe makolekole and his valimba group: A glimpse of the technique of the sena xylophone. Afr. Music, 7(1):82-104, 1991. doi: 10.21504/amj.v7i1.1932.

[21] Edward C. Carterette, Roger A. Kendall, and Sue Carole De Vale. Comparative acoustical and psychoacoustical analyses of gamelan instrument tones. Journal of the Acoustical Society of Japan (E), 14(6):383-396, 1993. doi: 10.1250/ast.14.383.

[22] Albrecht Schneider. Sound, pitch, and scale: From "tone measurements" to sonological analysis in ethnomusicology. Ethnomusicology, 45(3):489-519, 2001. doi: 10.2307/852868.

[23] K. Attakitmongcol, R. Chinvejkitvanich, and S. Sujitjorn. Characterization of traditional thai musical scale. In Proceedings of the 5th WSEAS International Conference on Acoustics and Music: Theory \& Applications (AMTA04), 2004.

[24] J. Zhang, X. Xiao, and Y. K. Lee. The early development of music. analysis of the jiahu bone flutes. Antiquity, 78(302): 769778, 2004. doi: 10.1017/S0003598X00113432.

[25] W. A. Sethares. Tuning, Timbre, Spectrum, Scale. Springer Science \& Business Media, 2005.

[26] G. Li. The effect of inharmonic and harmonic spectra in javanese gamelan tuning (1): A theory of the sléndro. In Proceedings of the 7th WSEAS International Conference on Acoustics \&3 Music: Theory \& A Applications, pages 65-71, Stevens Point, Wisconsin, USA, 2006. World Scientific and Engineering Academy and Society (WSEAS).

[27] L. E. McNeil and S. Mitran. Vibrational frequencies and tuning of the african mbira. J. Acoust. Soc. Am., 123(2):1169-1178, 2008. doi: $10.1121 / 1.2828063$.

[28] M. Kuss. Music in Latin America and the Caribbean: An Encyclopedic History: Volume 1: Performing Beliefs: Indigenous Peoples of South America, Central America, and Mexico. University of Texas Press, 2010.

[29] T. Wiggins. Tuning xylophones: Dagara instruments in the town of nandom, north-west ghana. Journal of Performing Arts, page $185,2011$.

[30] J. Garzoli. The myth of equidistance in thai tuning. Anal Approaches Music, 4(2):1-29, 2015. 
[31] N. Wisuttipat. Relative nature of thai traditional music through its tuning system. International Journal of Creative and Arts Studies, 2(1):86-97, 2015. doi: 10.24821/ijcas.v2i1.1441.

[32] A. Morkonr, S. Punkubutra, et al. The collecting process of xylophone's sound d (ranād xek) from art to numerical data. In 2018 International Conference on Engineering, Applied Sciences, and Technology (ICEAST), pages 1-4, 2018. doi: 10.1109/ ICEAST.2018.8434434.

[33] R. Bader. Temperament in tuning systems of southeast asia and ancient india. In Computational Phonogram Archiving, pages 75-107. Springer, 2019. doi: 10.1007/978-3-030-02695-0_3.

[34] C. M. L. Kimberlin. Masinqo and the Nature of Qanat. PhD thesis, 1976.

[35] F. Falceto and S. Weisser. Investigating qanat in amhara secular music: An acoustic and historical study. Annales d'thiopie, 28(1):299-322, 2013. doi: 10.3406/ethio.2013.1539.

[36] Peter Q. Pfordresher and Steven Brown. Vocal mistuning reveals the origin of musical scales. Eur. J. Cogn. Psychol., 29(1): 35-52, 2017. doi: 10.1080/20445911.2015.1132024.

[37] J. A. Siegel and W. Siegel. Categorical perception of tonal intervals: Musicians cant tell sharp from flat. Percept. Psychophys., 21(5):399-407, 1977. doi: 10.1037/h0094008.

[38] E. M. Burns and S. L. Campbell. Frequency and frequencyratio resolution by possessors of absolute and relative pitch: Examples of categorical perception? J. Acoust. Soc. Am., 96(5):2704-2719, 1994. doi: 10.1121/1.411447.

[39] J. Serra, G. K. Koduri, M. Miron, and X. Serra. Assessing the tuning of sung indian classical music. In ISMIR, pages 157-162, 2011.

[40] P. Larrouy-Maestri, P. M. C. Harrison, and D. Müllensiefen. The mistuning perception test: A new measurement instrument. Behav. Res. Methods, 51(2):663-675, 2019. doi: 10.3758/s13428-019-01225-1.

[41] B. Hagerman and J. Sundberg. Fundamental frequency adjustment in barbershop singing. Speech, Music and Hearing Quarterly Progress and Status Report, 21(1):28-42, 1980.

[42] M. Perlman and C. L. Krumhansl. An experimental study of internal interval standards in javanese and western musicians. Music Percept., 14(2):95-116, 1996. doi: 10.2307/40285714.

[43] J. H. McDermott, M. V. Keebler, C. Micheyl, and A. J. Oxenham. Musical intervals and relative pitch: Frequency resolution, not interval resolution, is special. J. Acoust. Soc. Am., 128(4):1943-1951, 2010. doi: 10.1121/1.3478785.

[44] P. E. Savage, S. Brown, E. Sakai, and T. E. Currie. Statistical universals reveal the structures and functions of human music. P. Natl. Acad. Sci. Usa., 112(29):8987-8992, 2015. doi: 10.1073/pnas.1414495112.

[45] Gerald J. Balzano. The group-theoretic description of 12-fold and microtonal pitch systems. Comput. Music J., 4(4):66-84, 1980. doi: $10.2307 / 3679467$.

[46] E. J. Kessler, C. Hansen, and R. N. Shepard. Tonal schemata in the perception of music in bali and in the west. Music Percept., 2(2):131-165, 1984. doi: 10.2307/40285289.

[47] E. G. Schellenberg and S. E. Trehub. Natural musical intervals: Evidence from infant listeners. Psychol. Sci., 7(5):272-277, 1996. doi: 10.1111/j.1467-9280.1996.tb00373.x.

[48] J. McDermott and M. Hauser. The origins of music: Innateness, uniqueness, and evolution. Music Percept., 23(1):29-59, 2005. doi: 10.1525/mp.2005.23.1.29.

[49] Barry Ross and Sarah Knight. Reports of equitonic scale systems in african musical traditions and their implications for cognitive models of pitch organization. Music. Sci., page 1029864917736105, 2017. doi: 10.1177/1029864917736105.

[50] A. T. Tierney, F. A. Russo, and A. D. Patel. The motor origins of human and avian song structure. P. Natl. Acad. Sci. Usa., 108(37):15510-15515, 2011. doi: 10.1073/pnas.1103882108.

[51] P. Harrison and M. Pearce. Simultaneous consonance in music perception and composition. Psychol. Rev., 2019.

[52] Richard Parncutt. Revision of terhardt's psychoacoustical model of the root(s) of a musical chord. Music Percept., 6(1):65-93, 1988. doi: $10.2307 / 40285416$.

53] Richard Parncutt and Hans Strasburger. Applying psychoacoustics in composition: "harmonic" progressions of "nonharmonic" sonorities. Perspect. New Music, 32(2):88-129, 1994. doi: 10.2307/833600.

[54] Richard Parncutt and Hans Strasburger. A Computational Model of the Cognition of Tonality. PhD thesis, 2013.

[55] Frieder Stolzenburg. Harmony perception by periodicity detection. J. Math. Music, 9(3):215-238, 2015. doi: 10.1080/ 17459737.2015 .1033024$.

[56] P. Harrison and M. T. Pearce. An energy-based generative sequence model for testing sensory theories of western harmony. arXiv preprint arXiv:180\%.00790, 2018.

[57] H. Schaffrath. The essen folksong collection, 1995.

[58] C. S. Sapp. Online database of scores in the humdrum file format. In ISMIR, pages 664-665, 2005.

[59] P. Van Kranenburg and M. de Bruin. The meertens tune collections: Mtc-fs-inst 2.0. Technical report, 2019.

60] S. Senturk. Computational modeling of improvisation in turkish folk music using variable-length markov models. Master's thesis, 2011 